\title{
Wahlkreisarbeit zahlt sich doppelt aus - Zur Wirkung des Amtsinhaberstatus einer Partei auf ihren Zweitstimmenanteil bei den Bundestagswahlen 1949 bis 1998
}

\author{
Jens Hainmueller, Holger Lutz Kern und Michael Bechtel*
}

\section{Einleitung}

Seit den frühen 90er Jahren haben mehr als 30 Länder Wahlsysteme eingeführt, die die Mehrheitswahl in Einerwahlkreisen mit Elementen der Verhältniswahl verbinden. Es vermag daher nicht zu überraschen, dass die wissenschaftliche Zurückhaltung, mit der solchen kombinierten Wahlsystemen anfangs vor dem Hintergrund der idealtypischen Unterscheidung zwischen Verhältnis- und Mehrheitswahlrecht begegnet wurde, inzwischen einem erheblichen Forschungsinteresse gewichen ist (Massicotte und Blais 1999; Shugart und Wattenberg 2001; Ferrara et al. 2005). Nicht nur die Entstehung solcher Wahlsysteme (Bawn 1993; Shugart 2001), sondern auch deren Bedeutung für strategisches Wählen und den Eintritt neuer Parteien in den politischen Wettbewerb wurden dabei ausgiebig untersucht (Bawn 1999; Gschwend et al. 2003; Moser und Scheiner 2005). Die Literatur hat inzwischen auch den Einfluss kombinierter Wahlsysteme auf das Verhalten des Gesetzgebers (Lancaster und Patterson 1990; Stratmann und Baur 2002; Bawn und Thies 2003) und die Entwicklung von Parteiensystemen (Shugart und Wattenberg 2001; Ferrara et al. 2005) analysiert.

Ein wichtiger Grund für das steigende Forschungsinteresse an kombinierten Wahlsystemen besteht darin, dass diese scheinbar die Möglichkeit bieten, Wählerverhalten unter den Bedingungen unterschiedlicher Wahlsysteme (Mehrheits- und Verhältniswahl) einem kontrollierten Vergleich zu unterziehen (Moser und Scheiner 2005: 260). Die Wirkung unterschiedlicher Wahlsysteme könne hier, so das gängige Argument, bei gleichzeitiger Kontrolle für Störvariablen untersucht werden (Lancaster und Patterson 1990; Stratmann und Baur

* Wir danken Barry Burden, Alexis Diamond, Federico Ferrara und Walter R. Mebane, Jr., den Teilnehmern des Political Behavior and Political Psychology Workshops an der Harvard University und den Teilnehmern des Strategic Voting Panels der 2006 Midwest Political Science Association Conference für hilfreiche Kommentare. 
2002; Moser 1999; Moser und Scheiner 2005). Dieses forschungslogische Argument kann aber nur dann überzeugen, wenn die beiden Komponenten des Wahlsystems tatsächlich unabhängig voneinander sind. Im Falle des deutschen Wahlsystems dürfen sich der Erst- und Zweitstimmenanteil einer Partei in einem Wahlkreis nicht gegenseitig beeinflussen. Neuere empirische Belege für die Existenz von so genannten Kontaminationseffekten, also von Wechselwirkungen zwischen den in kombinierten Wahlsystemen verwendeten Wahlrechtselementen, lassen diese entscheidende Voraussetzung jedoch höchst fragwürdig erscheinen (Herron und Nishikawa 2001; Cox und Schoppa 2002; Ferrara et al. 2005).

Unsere Arbeit baut auf diesen neueren Forschungsergebnissen auf und identifiziert erstmals die Parteizugehörigkeit eines Kandidaten, der seinen Wahlkreis bei der letzten Wahl gewonnen hat (Amtsinhaber), als eine wichtige Ursache von Kontamination. Falls ein guter Wahlkreisabgeordneter die Popularität seiner Partei im Wahlkreis erhöht, ist anzunehmen, dass dieser Kontaminationseffekt auch dann auftritt, wenn der Wahlkreisabgeordnete selbst nicht mehr zur Wiederwahl steht. Bislang wurde diese Wirkung des Amtsinhaberstatus von der Forschung zu Kontaminationseffekten übersehen. Dies mag daran liegen, dass sich die Kontaminationsforschung bisher darauf konzentriert hat, den Einfluss der bloßen Existenz von Wahlkreisbewerbern auf den Zweitstimmenerfolg einer Partei zu untersuchen. Den Befunden dieser Forschungsanstrengungen zufolge lohnt es sich für Parteien in jedem Fall, eigene Wahlkreisbewerber aufzustellen, da eigene Kandidaten aufstellende Parteien im Durchschnitt einen höheren Zweitstimmenanteil erhalten als Parteien, die auf die Aufstellung von eigenen Wahlkreisbewerbern verzichten.

In der umfangreichen Literatur zum Amtsinhaberbonus wird argumentiert, dass Wahlkreisabgeordnete einen Vorteil genießen, wenn sie in ihrem Wahlkreis erneut kandidieren. So haben beispielsweise in den Vereinigten Staaten und anderen Ländern Wahlkreisabgeordnete nachweislich einen bedeutenden Einfluss auf das Wahlergebnis in ihrem Wahlkreis (incumbency effects). Verbindet man diese beiden Literaturstränge, so ergibt sich folgendes Argument: Wirkt schon die bloße Präsenz eines Wahlkreiskandidaten positiv auf den Zweitstimmenanteil seiner Partei, so sollte der Amtsinhaberstatus, den dieser in seinem Wahlkreis genießt, eine noch weitaus stärkere Wirkung auf den Zweitstimmenanteil seiner Partei entfalten. Schließlich haben eine Partei und ihr Kandidat mit dem Gewinn eines Wahlkreises vier Jahre lang die Möglichkeit, die Wähler von den eigenen Vorteilen zu überzeugen. Dies ist ein weitaus längerer Zeitraum als der wenige Monate andauernde Wahlkampf, den die Literatur 
bislang als Ursache von Kontaminationseffekten ausgemacht hat. Wahlkreisabgeordnete müssten also nicht nur einen Vorteil im Hinblick auf ihren Erststimmenanteil genießen, sondern auch in der Lage sein, in ihrem Wahlkreis für ihre Partei eine höhere Zahl an Zweitstimmen zu erringen.

Zur Überprüfung dieser Hypothese eignen sich Bundestagswahlergebnisse in besonderem Maße, denn mit seinem personalisierten Verhältniswahlrecht verfügt Deutschland über das älteste kombinierte Wahlsystem der Welt. Die deutschen Bürger hatten somit ausreichend Zeit und Gelegenheit, sich mit den Möglichkeiten strategischen Wählens vertraut zu machen (Nohlen 2004; Bawn 1999). Wenn Kontaminationseffekte in kombinierten Wahlsystemen tatsächlich existieren, so sollten sie hier zu finden sein. Zur empirischen Überprüfung verwenden wir eine Regressionsdiskontinuitätsanalyse (RD-Analyse). Dieser Ansatz hilft uns, jene inferentiellen Hindernisse zu überwinden, die zuverlässige Kausalschlüsse in nicht-experimentellen Studien erschweren. Die RD-Analyse nutzt die annähernd randomisierte Zuweisung des Amtsinhaberstatus, die immer dann auftritt, wenn der Wahlausgang in einem Wahlkreis knapp ist. In solchen Fällen sind, verglichen mit dem in der Wahlforschung weit verbreiteten Standard-Regressionsmodell, die für Schlüsse auf kausale Effekte erforderlichen Annahmen weitaus schwächer. Die empirischen Ergebnisse zeigen, dass der Amtsinhaberstatus einer Partei in Bundestagswahlen den Zweitstimmenanteil im Wahlkreis um durchschnittlich 1 bis 1,5 Prozentpunkte erhöht. Mit Hilfe von Simulationen stellen wir darüber hinaus fest, dass Effekte dieser Größenordnung ausreichend sind, um politisch bedeutsame Verschiebungen in der Sitzverteilung des Bundestages auszulösen.

Im Folgenden gehen wir zunächst auf Vorarbeiten im Bereich der Forschung zu Kontaminationseffekten in kombinierten Wahlsystemen ein und legen sodann unser theoretisches Argument dar. Der dritte Abschnitt stellt unser statistisches Modell sowie die verwendeten Daten vor. Abschnitt vier präsentiert die empirischen Befunde, denen im fünften Abschnitt die Ergebnisse der Sensitivitätsanalyse folgen. Die Arbeit schließt mit einer Zusammenfassung unserer zentralen Ergebnisse und ihren Implikationen für die weitere Forschung sowie das strategische Verhalten von Parteien im deutschen Parteienwettbewerb. 


\section{Kontamination und der Amtsinhaberstatus von Parteien}

Kontaminationseffekte können in zwei Richtungen wirken. ${ }^{1}$ Die Existenz von Einerwahlkreisen kann zum einen den Stimmenwettbewerb im Verhältniswahlelement beeinflussen. Inspiriert von den in Deutschland gemachten Erfahrungen mit einem kombinierten Wahlsystem bemerkt zum Beispiel Maurice Duverger, dass der für Einerwahlkreise typische Zweiparteienwettbewerb auch auf die Ebene des Verhältniswahlelements übergreift (Duverger 1986: 72). Zum anderen ist die umgekehrte Wirkungsrichtung, also vom proportionalen hin zum majoritären Wahlsystemelement, denkbar. Cox und Schoppa (2002: 1031) argumentieren beispielsweise, dass die in Einerwahlkreisen vermutete starke Tendenz zur Ausbildung eines Zweiparteiensystems (Duverger 1954; Cox 1997) in den kombinierten Wahlsystemen Deutschlands, Japans und Italiens erheblich abgeschwächt wird. Denn im Unterschied zu reinen Mehrheitswahlsystemen besteht hier für kleine Parteien auch dann ein Anreiz, sich im Einerwahlkreis zur Wahl zu stellen, wenn sie kaum Aussichten auf Erfolg haben, ,in order to give their party a human face that they can use to boost the party's [PR] vote totals“ (Cox und Schoppa 2002: 1031). Eine solche Strategie erklärt auch den Befund von Herron und Nishikawa (2001), die feststellen, dass die Anzahl der Parteien in den Einerwahlkreisen der gemischten Wahlsysteme Russlands und Japans größer ist als in reinen Mehrheitswahlsystemen. Mit der Aufstellung von Kandidaten in möglichst vielen Wahlkreisen - selbst wenn diese keine Chance haben, ihren Wahlkreis zu gewinnen - können kleine Parteien für das eigene Programm werben und so ihren Stimmenanteil im Verhältniswahlelement erhöhen. Zu einem ähnlichen Ergebnis kommt auch die Untersuchung einer größeren Anzahl kombinierter Wahlsysteme durch Ferrara et al. (2005: Kapitel 3). Diese Studie zeigt, dass der Stimmenanteil einer Partei im proportionalen Wahlsystemelement positiv mit der Aufstellung eigener Wahlkreisbewerber korreliert.

Mittels der bislang gemachten Befunde ist die Kontaminationsforschung zu neuen Einsichten in die Funktionsweise von kombinierten Wahlsystemen gelangt. Allerdings sind die bisherigen Ergebnisse nicht gegen methodische Kritik gefeit. So lässt sich einwenden, dass Parteien als rationale Akteure einen eige-

1 Der Ausdruck Kontamination wird Ferrara et al. entsprechend definiert: "Contamination is present, at the micro-level, when the behavior of a voter, a party, a candidate, or a legislator in one tier of the election is demonstrably affected by the institutional rules employed in the other tier. At the aggregate level, contamination is observed when a particular outcome produced in one tier (like the number of parties) is affected by the institutional features of the other tier" (2005: 8-9). 
nen Kandidaten vor allem in denjenigen Wahlkreisen ins Rennen schicken werden, in denen sie ohnehin ein überdurchschnittliches Ergebnis erwarten oder in denen die organisatorischen Ressourcen für einen erfolgreichen Wahlkampf zur Verfügung stehen. Selbst wenn diese Vermutung nur zum Teil zuträfe, handelte es sich damit bei dem beobachteten Zusammenhang zwischen der Präsenz eines eigenen Wahlkreisbewerbers und einem höheren Zweitstimmenanteil um eine Scheinkorrelation. Dieser Gefahr des Bias durch Selbstselektion ist man sich bei der Erforschung von Kontaminationseffekten sehr wohl bewusst. Nicht umsonst versuchen die soeben zusammengefassten Studien, der Verzerrung ihrer Schätzungen durch die Aufnahme von Kontrollvariablen entgegenzuwirken. So verwenden Cox und Schoppa (2002: 1034) den in der Vergangenheit erzielten Stimmenanteil einer Partei im Wahlkreis als Messinstrument für die Popularität einer Partei. Herron und Nishikawa (2001) fügen ihrem Modell eine binäre Indikatorvariable für den Amtsinhaberstatus hinzu und nehmen außerdem im Falle Japans für einige Parteien deren Stimmenanteil in den vergangenen Wahlen zum Oberhaus in ihre Regressionsgleichung auf. In einer jüngeren Arbeit (Ferrara et al. 2005) wird der Amtsinhaberstatus als Proxy für Popularität verwendet; außerdem werden die statistischen Modelle auch um einige demographische und regionale Kontrollvariablen erweitert. Interessanterweise zeigen die Ergebnisse dieser Regressionsanalysen, dass Amtsinhaberschaft einen positiven und signifikanten Effekt auf den Stimmenanteil im proportionalen Wahlsystemelement besitzt. Allerdings degradiert die Arbeit von Ferrara et al. (2005) den Amtsinhaberstatus einer Partei zu einer reinen Kontrollvariablen für die Popularität einer Partei, weshalb den theoretischen Implikationen dieses Befundes keinerlei Beachtung geschenkt wird. Im Unterschied hierzu ist die Amtsinhaberschaft einer Partei in unserer Arbeit der zentrale, kausal wirksame Faktor. Wir bezeichnen die Amtsinhaberschaft einer Partei, also die Tatsache, dass ihr Kandidat in seinem Wahlkreis das Direktmandat erringen konnte, deshalb im Folgenden als Treatment. Unser Forschungsdesign verfolgt das Ziel, den kausalen Effekt dieses Treatments zu bestimmen.

Darüber hinaus ist festzuhalten, dass die existierenden Vorarbeiten auf einer sehr starken statistischen Annahme - die Zuweisung des Amtsinhaberstatus (bei Kontrolle für Störvariablen) unabhängig von den potentiellen Wahlergebnissen $^{2}$ ist (unconfounded treatment assignment) (Rubin 1974; Rosenbaum 2002) - ruhen. Anders ausgedrückt lautet diese grundlegende Annahme: Für

2 Potentielle Wahlergebnisse sind hier als potential outcomes nach Rubins Modell der Kausalen Inferenz zu verstehen (siehe hierzu die ausführliche Darstellung im dritten Abschnitt dieser Arbeit). 
jede Partei ist unter Berücksichtigung ihres zuletzt erzielten Stimmenanteils bzw. anderer regionaler oder sozi-demographischer Kontrollvariablen die Wahrscheinlichkeit, in einem Wahlkreis einen eigenen Kandidaten aufzustellen, über treated und untreated Wahlkreise hinweg die gleiche (das heißt, Wahlkreise in denen dann tatsächlich ein eigener Kandidat antritt oder nicht antritt). Dies ist offensichtlich eine übermäßig restriktive Annahme, impliziert sie doch, dass für alle Störvariablen kontrolliert wird. Störvariablen sind dabei alle Faktoren, die sowohl die Zuweisung des Amtsinhaberstatus als auch die potentiellen Wahlergebnisse beeinflussen.

Die Regressionsdiskontinuitätsanalyse (RD-Analyse) erlaubt es uns, den kausalen Effekt des Amtsinhaberstatus zu ermitteln, ohne auf derartige Annahmen angewiesen zu sein. Die RD-Analyse macht sich dabei die quasirandomisierte Zuweisung des Amtsinhaberstatus zu nutze, die immer dann auftritt, wenn ein Wahlkreis knapp gewonnen oder verloren wurde. Geht man davon aus, dass Parteien ihren Stimmenanteil nie exakt vorhersagen können, sollten äußerst knapp gewonnene Wahlkreise hinsichtlich der Verteilung (unbeobachteter) Störvariablen denen gleichen, die von derselben Partei äußerst knapp verloren wurden. Wie später noch gezeigt werden wird, liegt damit ein natürlich auftretendes Kontrafaktum vor, welches unter vergleichsweise schwachen Annahmen die Schätzung kausaler Effekte ermöglicht.

Die bisherigen Forschungsergebnisse im Bereich der Kontaminationsforschung werden durch ein weiteres, nicht minder beachtenswertes methodisches Problem in Frage gestellt. Die Schätzung von durch die Gegenwart von Wahlkreisbewerbern verursachten Kontaminationseffekten ist nämlich nur dann möglich, wenn Parteien nicht in allen Wahlkreisen mit einem eigenen Kandidaten vertreten sind. In den meisten Fällen ist diese Voraussetzung jedoch nicht gegeben, da Parteien so gut wie immer einen eigenen Wahlkreisbewerber aufstellen. Diese Tatsache wird in der Literatur als Beleg dafür angesehen, dass Parteien sich der Existenz von Kontaminationseffekten bewusst sind und diese als strategisch handelnde Akteure gezielt zu nutzen wissen (Cox und Schoppa 2002). Ohne Varianz in der unabhängigen Variablen ist eine Schätzung des kausalen Effektes jedoch unmöglich. ${ }^{3}$ Für unsere Arbeit besteht dieses Problem nicht, da

3 Cox und Schoppa (2002) präsentieren deshalb nur für Wahlen in Japan empirische Schätzungen und Ferrara et al. (2005) beschränken ihre Ausführungen zu Deutschland auf die Bundestagswahl 1953. Angemerkt wird dabei lediglich, dass in späteren Bundestagswahlen sogar kleine Parteien wie die FDP oder die Grünen fast in jedem Wahlkreis mit einem eigenen Kandidaten vertreten waren. Dies ist nicht verwunderlich, zumal der Anreiz gerade für kleine Parteien, einen eigenen Wahlkreisbewerber aufzustellen, mit der Wahlrechtsreform von 1956 erheblich verstärkt wurde. War es nämlich vormals noch ausreichend gewesen, ein Direktmandat zu er- 
unsere zentrale Treatment-Variable, der Amtsinhaberstatus einer Partei, sowohl über Wahlkreise als auch über Zeit hinweg variiert.

Warum aber sollte sich der Amtsinhaberstatus einer Partei, also die Tatsache, dass ihr Kandidat den Wahlkreis in der letzten Wahl gewinnen konnte, positiv auf ihren Zweitstimmenanteil auswirken? Zur Beantwortung dieser Frage verknüpfen wir die fast schon klassische Literatur zu den Vorteilen von Amtsinhabern, die auf Untersuchungen des Verhaltens von Wahlkreisabgeordneten aufbaut, mit den neueren Arbeiten zu Kontaminationseffekten in kombinierten Wahlsystemen.

In der Forschung zum Amtsinhaberbonus (incumbency advantage) konnte nachgewiesen werden, dass erneut kandidierende Wahlkreisabgeordnete einen erheblichen Vorteil genießen. Für die Vereinigten Staaten hat die Literatur einige spezifische Ursachen des Amtsinhaberbonus in Kongresswahlen ausmachen können. Hierzu gehören beispielsweise die strategische Entscheidung von Wahlkreisabgeordneten, sich nicht zur Wiederwahl zu stellen, wenn der Erfolg unsicher ist oder das Kalkül neuer Kandidaten, den bisherigen Abgeordneten nur dann herauszufordern, wenn er politisch bereits angeschlagen erscheint. Der vermutlich auf ihre starke Medienpräsenz zurückzuführende hohe Bekanntheitsgrad amerikanischer Kongressabgeordneter wird ebenfalls als Erklärung angeführt. Auch die Fähigkeit eines Wahlkreisabgeordneten, sich erfolgreich um öffentliche Gelder für den eigenen Wahlkreis zu bemühen (pork barrel projects) sowie die Bürger im Umgang mit der Verwaltung zu unterstützen, wird als Quelle des incumbency advantage betrachtet. Diese Leistungen - so die Theorie - honorieren die Wähler mit ihren Stimmen. ${ }^{4}$

Während die deutsche Politikwissenschaft den Parteien erhebliches Forschungsinteresse entgegengebracht hat, wurde Kandidaten und Abgeordneten vergleichsweise wenig Aufmerksamkeit geschenkt. Insbesondere das Verhalten von direkt in den Bundestag gewählten Abgeordneten in ihren Wahlkreisen ist bislang wenig erforscht. Selbst die gängige Diskussion um eine zunehmende Personalisierung der Politik (Brettschneider 2002; Pappi und Shikano 2001) bezieht sich vornehmlich auf den Wettlauf der Spitzenkandidaten um das Amt des Bundeskanzlers, kaum jedoch auf einzelne Abgeordnete. Dies erscheint

ringen, um im Bundestag vertreten zu sein, wurde diese Zahl nun auf drei heraufgesetzt. Der Gesetzgeber hat damit den Weg ins Parlament über den Gewinn von Direktmandaten für kleine Parteien so gut wie aussichtslos werden lassen (vergleiche Nohlen 2004: 306).

4 Siehe Cox (2002); Ansolabehere, Snyder, und Stewart (2000); Ansolabehere und Steward (2004); Levitt und Wolfram (1997); King und Gelman (1990) sowie die dort diskutierte klassische Literatur. 
umso überraschender angesichts der Tatsache, dass die personalisierte Verhältniswahl bewusst eingeführt wurde, um die persönliche Verbindung zwischen Bürgern und Wahlkreisabgeordneten und damit die Repräsentation lokaler Anliegen zu stärken (Nohlen 2004). Die Frage, ob auch deutsche Wahlkreisabgeordnete wie ihre amerikanischen und britischen Kollegen von einem Amtsinhaberbonus hinsichtlich ihres Erststimmenanteils profitieren, ist weitgehend unbeachtet geblieben. Lediglich Bawn (1999) sowie Moser und Scheiner (2005) stellen einen kleinen Amtsinhaberbonus bei Bundestagswahlen fest, stützen sich dabei aber allein auf den Unterschied zwischen Erst- und Zweitstimmen als Maß für kandidatenbezogene Stimmen. Sollten Kontaminationseffekte existieren was von den bisherigen Forschungsergebnissen nahegelegt wird - führt eine solche Messtrategie auf Grund der oben ausgeführten Schwierigkeiten jedoch zu verzerrten Ergebnissen.

Auch die Frage, ob Wahlkreiskandidaten einen Einfluss auf den Zweitstimmenanteil ihrer Parteien haben, harrt noch einer Antwort. Der Literatur zu Kontaminationseffekten nach hat schon die bloße Präsenz eines Wahlkreisbewerbers eine positive Wirkung auf den Zweitstimmenanteil seiner Partei (Cox und Schoppa 2002; Herron und Nishikawa 2001; Ferrara et al. 2005). Cox und Schoppa zufolge stellen kleinere Parteien auch in aussichtslosen Wahlkreisen einen eigenen Kandidaten auf, um den Wählern ihr Politikangebot ins Gedächtnis zu rufen und dadurch ihren Zweitstimmenanteil zu erhöhen. Inwieweit dies auch für Deutschland zutrifft, ist bislang unklar. Falls aber die bloße Aufstellung eines eigenen Wahlkreisbewerbers tatsächlich zu einem höheren Zweitstimmenanteil führt, dann sollten Wahlkreisabgeordnete - neben dem Vorteil, den sie im Hinblick auf ihren Erststimmenanteil genießen - auch die Fähigkeit besitzen, ihrer Partei eine zusätzliche Anzahl Zweitstimmen zu sichern. Da die bisher festgestellten Kontaminationseffekte aufgrund der Präsenz von Wahlkreisbewerbern bereits nach der vergleichsweise kurzen Wahlkampfzeit auftreten, ist davon auszugehen, dass der Amtsinhaberstatus einer Partei eine mindestens ebenso große Wirkung auf ihren Zweitstimmenanteil hat. Schließlich eröffnet der Sieg im Wahlkreis einem Abgeordneten und seiner Partei vier Jahre lang die Möglichkeit, die Wähler von den eigenen Qualitäten zu überzeugen. Dies ist natürlich ein weitaus längerer Zeitraum als die wenige Monate andauernde Wahlkampfzeit, welche die Literatur bislang als Ursache von Kontaminationseffekten ausgemacht hat. Vor diesem Hintergrund betrachtet sollte der positive Effekt der Parteizugehörigkeit eines Amtsinhabers also noch größer sein als jener, der durch das Aufstellen eines eigenen Wahlkreisbewerbers ausgelöst wird. 
Was sind nun die Kausalmechanismen, die einen positiven Effekt des Amtsinhaberstatus auf den Zweitstimmenanteil erwarten lassen? Umfragen unter Bundestagsabgeordneten weisen nach, dass diese einen beträchtlichen Teil ihrer Zeit in ihren Wahlkreisen verbringen. Die Wahlkreisarbeit umfasst neben den unvermeidlichen Repräsentationsaufgaben (Schützenfeste und 80. Geburtstage) im Wesentlichen zwei Aufgabenkomplexe. Zum einen ist da die Vertretung der Anliegen des Wahlkreises gegenüber Land und Bund. Im Vordergrund steht dabei die Einflussnahme auf Bundes- und Landesgesetzgebung sowie die Vertretung von Wahlkreisinteressen gegenüber Bundes- und Landesministerien. Der bereits von Zeuner (1970: 118) konstatierte Mangel an Arbeiten, die untersuchen, in welchem Umfang Wahlkreisabgeordnete die Interessen ihres Wahlkreises vertreten können und dies auch tatsächlich tun, scheint bis heute nicht beseitigt worden zu sein. Belegt ist lediglich, dass Bundestagsabgeordnete selbst eine effektive Vertretung der Interessen ihres Wahlkreises als entscheidenden Faktor für die angestrebte Wiederwahl betrachten (Lancaster und Patterson 1990; Patzelt 1993). Solche Aussagen werden durch die Studie von Stratmann und Baur (2002) bestätigt. Diese weist nach, dass durch Direktwahl und nicht über Listenplätze in den Bundestag gelangte Abgeordnete ihre Mitgliedschaften in Parlamentsausschüssen so wählen, dass sie die Interessen ihrer Wahlkreise besser vertreten können.

Zum anderen nehmen sich Wahlkreisabgeordnete aber auch der unterschiedlichsten Anliegen einzelner Bürger an. Ein Abgeordneter fasst dies so zusammen: „Also ich würde zunächst einmal sagen: es gibt nichts, was es nicht gibt. Ich habe in all meinen Sprechstunden die unmöglichsten Punkte - von Nachbarstreiten, Problemen häuslicher Art bis zu Fragen der Renten-, der Versetzungs-, der Beförderungswünsche u.ä. - mit auf den Tisch bekommen. Mir ist eigentlich nicht bekannt, dass es irgendwas nicht gegeben hätte. [...] An den Politiker, sprich den verantwortlichen Mandatsträger, wendet man sich in der Hoffnung, er kann etwas bewegen - für mich, für das Anliegen. Die Verwaltung ist für viele sehr anonym. Hier hat sich der Parlamentarier weg von der legislativen Funktion hin zur Anlaufstelle für alles entwickelt" (zitiert nach Patzelt 1993: 367).

Für die Bürger ansprechbar zu sein und ihnen mit ihren Anliegen zu helfen ist also ein weiterer wichtiger Teil der Wahlkreisarbeit. Leider gibt es keine systematischen Untersuchungen, die Auskunft darüber geben, inwieweit Wähler Wahlkreisabgeordnete tatsächlich für guten „Wählerservice“ belohnen. Eine Ausnahme ist die Studie von Klingemann und Wessels (2001), die zu dem Schluss kommt, dass das Wahlverhalten in der Bundestagswahl 1998 stark von 
den Eindrücken geprägt war, die Wähler von der Qualität der Wahlkreisarbeit ihrer Wahlkreisabgeordneten hatten. Falls dieser Zusammenhang existiert, stellt sich natürlich die Frage, inwieweit sich die besondere Popularität bestimmter Wahlkreisabgeordneter auf den Stimmenanteil ihrer Parteien im proportionalen Wahlsystemelement niederschlägt. Die Tatsache, dass Parteigremien bei der Kandidatenaufstellung auch auf die Qualität der Wahlkreisarbeit achten, spricht für einen solchen Zusammenhang (Zeuner 1970: 144-146; Patzelt 1993: 366382). Falls ein guter Wahlkreisabgeordneter auch die Popularität seiner Partei im Wahlkreis erhöht, ist anzunehmen, dass dieser Kontaminationseffekt selbst dann auftritt, wenn der Wahlkreisabgeordnete selbst nicht mehr zur Wiederwahl steht. Aus diesem Grund konzentrieren wir uns hier auf den Amtsbonus der Partei.

\section{Zum Verfahren der Regressionsdiskontinuitätsanalyse}

Wir verwenden eine Regressionsdiskontinuitätsanalyse (RD-Analyse), um den kausalen Effekt der Amtsinhaberschaft einer Partei auf zwei abhängige Variablen zu ermitteln: Den in der nächsten Wahl erzielten Stimmenanteil der Partei im proportionalen Element des Wahlsystems (Zweitstimmen) und das Abschneiden des parteieigenen Kandidaten im majoritären Element (Erststimmen). Als quasi-experimenteller Ansatz erlaubt die RD-Analyse kausale Effekte immer dann zu schätzen, wenn die Zuweisung zur Experimentalgruppe unstetig als Funktion einer oder mehrer Hintergrundvariablen erfolgt.

Die erste publizierte Anwendung der RD-Analyse ist die klassische Studie von Thistlethwaite und Campbell (1960). Untersucht wird darin die Wirkung von Stipendien auf die berufliche Karriere der Stipendienbewerber. Über die Vergabe der Stipendien wurde auf Basis eines schriftlichen Tests entschieden. Thistlethwaite und Campbell vergleichen eine Gruppe von Studenten, in welcher die Testergebnisse knapp unterhalb der erforderlichen Punktezahl lagen (Kontrollgruppe) mit einer anderen Gruppe von Studenten, in der die Testergebnisse knapp über der erforderlichen Punktezahl lagen (Experimentalgruppe). In unmittelbarer Nähe des über die Einteilung in eine der beiden Gruppen entscheidenden Schwellenwertes ist die Zuweisung des Treatments, also die Gewährung eines Stipendiums, so gut wie zufällig. Vom Glück im Stich gelassene Studenten, die den Test ganz knapp nicht bestanden, sollten denen, die nur wenige Punkte über der erforderlichen Punktezahl lagen, gleichen. Der einzige systematische Unterschied zwischen den Individuen in beiden Gruppen besteht 
darin, dass die mit Glück gesegneten Studenten ein Stipendium (das Treatment) erhielten und die anderen nicht. Thistlethwaite und Campbell steht so ein für kausale Inferenz nutzbares Kontrafaktum zur Verfügung.

Ein Prozess der Datengenerierung, der die Zuweisung zur Experimentalgruppe allein vom Überschreiten eines Schwellenwertes einer Hintergrundvariablen abhängig macht, tritt in der Realität erstaunlich häufig auf. Seit der wegweisenden Arbeit von Thistlethwaite und Campbell sind RD-Analysen deshalb häufig in so unterschiedlichen Wissenschaftsbereichen wie der Medizin, Ökonomie oder Soziologie zur Anwendung gekommen. ${ }^{5}$ Neuere Arbeiten aus der Ökonometrie sowie der Programmevaluation belegen außerdem ein steigendes Interesse an der Verwendung dieses Verfahrens. ${ }^{6}$ Die erste Anwendung der RDAnalyse im Bereich der Wahlforschung ist eine Studie des Ökonomen David Lee (im Erscheinen), welche den Amtsinhaberbonus in amerikanischen Kongresswahlen untersucht. Butler und Butler (2005) widmen sich unter Verwendung der RD-Analyse dem Verhalten von Senatsabgeordneten in den Vereinigten Staaten. Sieht man von diesen beiden Studien einmal ab, so ist festzustellen, dass Politikwissenschaftler das Potential der RD-Analyse als Instrument für kausale Inferenzen in nicht-experimentellen Studien bislang ignoriert haben.

Um die Grundidee der RD-Analyse darzustellen, eignet sich in besonderem Maße Rubins Modell der kausalen Inferenz. Rubin (1974; 1978; 1990; Holland 1986; Rosenbaum 2002) konzeptualisiert kausale Inferenz unter Rückgriff auf potentielle Ergebnisse (potential outcomes). Zur Darstellung dieses Ansatzes sind zunächst einige Definitionen nötig.

Wir bezeichnen $T V S_{i, j, t}$ als den ,wahren“ Stimmenanteil der Partei $j$ im Wahlkreis $i$ in der Wahl zum Zeitpunkt $t^{7}$ Dabei gehen wir davon aus, dass Partei $j$ zu den beiden Zeitpunkten $t$ und $t-1$ zur Wahl steht. ${ }^{8}$ Der tatsächlich erreichte Stimmenanteil der Partei $j$ wird durch die Variable $O V S_{i, j, t}$ erfasst. Sie ist das Ergebnis zweier Summanden:

5 Shadish et al. (2002: 208) bieten eine Übersicht neuerer Anwendungen. Siehe auch Trochim (1984) für weitere Beispiele und eine gute Einführung in die RD-Analyse.

6 Als theoretische Arbeiten zur Identifikation und Schätzung von RD-Analysen sind zu nennen: Hahn, Todd, und van der Klaauw (2001); Porter (2002); Battistin und Rettore (2002; 2003); Lee und Card (2004); Lee (im Erscheinen). Aktuelle empirische Anwendungen sind unter anderem Angrist und Lavy (1999); Black (1999); Berk und de Leeuw (1999); Lee et al. (2004); DiNardo und Lee (2004); Martorell (2004); Matsudaira (2004).

7 Um die Notation zu vereinfachen, wird an dieser Stelle auf die Unterscheidung zwischen Erstund Zweitstimmen verzichtet. Die Formeln für beide abhängigen Variablen sind identisch.

8 Es handelt sich dabei um eine harmlose Annahme, da SPD wie auch CDU/CSU bei Bundestagswahlen in allen Wahlkreisen antreten. 
$O V S_{i, j, t}=T V S_{i, j, t}+\eta_{i, j, t}$

$T V S_{i, j, t}$ ist eine systematische Komponente. Sie ist eine Funktion der Eigenschaften und Handlungen einer Partei (zum Beispiel ihr Wahlprogramm oder die unternommenen Wahlkampfanstrengungen) bzw. ihrer Vertreter (zum Beispiel die fachliche Kompetenz und Persönlichkeit des Wahlkreisbewerbers). $\eta_{i, j, t}$ hingegen ist eine exogene Zufallsvariable (wie beispielsweise das Wetter am Wahltag) mit einem Mittelwert von 0 und stetiger Dichte. Auf letzteres gehen wir weiter unten näher ein.

Wir definieren die Wirkung des Amtsinhaberstatus einer Partei als kausalen Effekt der Amtsinhaberschaft in einem Wahlkreis auf den Erst- bzw. Zweitstimmenanteil in der nächsten Wahl. $D_{i, j, t}$ ist ein binärer Indikator für den Treatment-Status, der den Wert 1 annimmt, wenn Partei $j$ der Amtsinhaber in einem Wahlkreis ist und 0, wenn dies nicht der Fall ist. Im Mehrheitswahlrecht ist der Treatment-Status (also die Zugehörigkeit zur Experimental- bzw. Kontrollgruppe) allein davon abhängig, ob bei der Wahl zum Zeitpunkt $t-1$ der Stimmenanteil eines Wahlkreisbewerbers im Wahlkreis $i$ größer war als der seines stärksten Gegners. Um den Abstand der Erststimmen (margin of victory) zu berechnen, ordnen wir für jeden Wahlkreis die Parteien entsprechend des von ihrem Kandidaten bei der Wahl $t-1$ erzielten Erststimmenanteils. Sei $W$ die Partei, deren Kandidat die meisten Stimmen gewonnen hat und $W-1$ die Partei, deren Kandidat den zweitgrößten Stimmenanteil vorweisen kann. Dann ergibt sich der Erststimmenabstand der siegreichen Partei bezogen auf den der zweitplatzierten als

$M V_{i, j, t-1}=O V S_{i, j, t-1}-O V S_{i, W-1, t-1}$.

Für jede andere Partei ist der Stimmenabstand zur siegreichen Partei

$M V_{i, j, t-1}=O V S_{i, j, t-1}-O V S_{i, w-1, t-1}$.

Der so berechnete Stimmenabstand ist positiv für eine Partei, wenn deren Kandidat den Wahlkreis für sich gewinnen konnte und negativ, wenn er gescheitert ist. Der Schwellenwert, den es für den Sieg nach Erststimmen zu überschreiten gilt, genannt $\bar{M} V$, ist damit 0 . Definiert man den Stimmenabstand $M V$ wie in Gleichung 2 geschehen, wird schnell einsichtig, wie der kausale Effekt der Amtsinhaberschaft einer Partei in einer RD-Analyse berechnet werden kann. 
Die Zuweisung zum Treatment hängt ja allein davon ab, ob der Stimmenabstand einer Partei in der vorhergehenden Wahl $\left(M V_{t-1}\right)$ den kritischen Wert 0 überschritten hat. Somit ist $D_{t}=1\left[M V_{t-1} \geq 0\right]$, wobei 1[ ] eine Indikatorfunktion darstellt, die den Wert 1 annimmt, wenn die innerhalb der eckigen Klammern formulierte Bedingung wahr ist und 0 gleicht, wenn dies nicht der Fall ist. ${ }^{9}$

Nun werden noch die Variablen $Y_{1, i, j}$ und $Y_{0, i, j}$ benötigt. $Y_{1, i, j}$ bezeichnet das potentielle Wahlergebnis der Partei $j$ im Wahlkreis $i$, wenn sie dem Treatment (Amtsinhaberstatus) ausgesetzt war und somit der Experimentalgruppe angehört. $Y_{0, i, j}$ steht für das potentielle Wahlergebnis der Partei $j$ im Wahlkreis $i$, wenn sie dem Treatment nicht ausgesetzt war und sich deshalb in der Kontrollgruppe befindet. Die beiden abhängigen Variablen sind der Erst- und Zweitstimmenanteil einer Partei in der folgenden Bundestagswahl.

Leider ist es ist nie möglich, für eine Einheit $i$ beide potentiellen Ergebnisse $Y_{1, i, j}$ und $Y_{0, i, j}$ zu beobachten. Beobachtbar ist nur das realisierte Ergebnis $Y_{i, j}=D_{i, j} \times Y_{1, i, j}+\left(1-D_{i, j}\right) \times Y_{0, i, j}$. Mit anderen Worten: Ist eine Partei Amtsinhaber in einem Wahlkreis, dann ist es unmöglich, ihren Anteil an Erst- und Zweitstimmen bei der nächsten Wahl zu beobachten, den sie erzielt hätte, wenn sie nicht der Amtsinhaber gewesen wäre (und umgekehrt). Daraus folgt, dass kausale Effekte niemals für eine einzelne Einheit geschätzt werden können. Paul W. Holland (1986) sieht in dieser Nicht-Beobachtbarkeit des Kontrafaktums das fundamentale Problem jeder Kausalanalyse. Allerdings ist es unter bestimmten Voraussetzungen möglich, den durchschnittlichen Treatment-Effekt (average treatment effect) $A T E=E\left[Y_{1}-Y_{0}\right]$ oder den durchschnittlichen Treatment-Effekt für die der Experimentalgruppe zugeordneten Einheiten (average treatment effect for the treated) $A T T=E\left[Y_{1}-Y_{0} \mid D=1\right] \mathrm{zu}$ ermitteln.

Die Stärke der RD-Analyse beruht auf der genauen Kenntnis des Mechanismus, der über die Zuweisung zur Experimental- und Kontrollgruppe entscheidet (assignment mechanism). Schon unter sehr schwachen Annahmen kann so der $A T E$ an jenem Schwellenwert bestimmt werden, der über die Zuweisung des Treatments entscheidet. Ergebnissen eines solchen Quasi-Experiments kann fast so viel Vertrauen entgegengebracht werden wie kausalen Schlüssen, die auf einem echten randomisierten Experiment basieren. Sind die Dichtefunktion der Zuweisungsvariablen (in unserem Fall $M V_{t-1}$ ) innerhalb der Trägermenge ebenso wie $E\left[Y_{0}\right]$ in unmittelbarer Umgebung des Schwellenwertes, der über die

9 In den verwendeten Daten gibt es keine Patt-Situation, der kritische Wert von 0 für $\bar{M} V$ wird also nie exakt erreicht, sondern immer unter- oder überschritten. 
Zuweisung des Treatments entscheidet, stetig, wird der ATE am Schwellenwert identifiziert: ${ }^{10}$

$$
\begin{aligned}
A T E & =E\left[Y_{1}-Y_{0} \mid M V_{t-1}=\bar{M} V_{t-1}\right] \\
& =E\left[Y \mid M V_{t-1}=\bar{M} V_{t-1}\right]-\lim _{\varepsilon \rightarrow 0} E\left[Y_{0} \mid M V_{t-1}=\bar{M} V_{t-1}-\varepsilon\right] \\
& =E\left[Y \mid M V_{t-1}=\bar{M} V_{t-1}\right]-\lim _{\varepsilon \rightarrow 0} E\left[Y \mid M V_{t-1}=\bar{M} V_{t-1}-\varepsilon\right]
\end{aligned}
$$

Die Annahme für die Identifikation des $A T E$ lautet, dass $E\left[Y_{0} \mid M V_{t-1}\right]$ an der Stelle $\bar{M} V_{t-1}$ stetig ist, also keine Sprungstelle vorliegt. ${ }^{11}$ Mittels dieser Annahme kann jede Unstetigkeit in unmittelbarer Umgebung des kritischen Wertes auf die Wirkung des Treatments zurückgeführt werden. Die Annahme impliziert weiterhin, dass bei ausreichend kleinem $\varepsilon$ die Wahlkreise mit marginaler Überschreitung des Schwellenwerts ein valides Kontrafaktum für diejenigen Wahlkreise darstellen, in denen der Schwellenwert nur knapp unterschritten wurde. Allerdings ist der so ermittelte $A T E$ rein lokaler Natur, da die Identifikation des kausalen Effekts ja ausschließlich anhand der Wahlkreise geschieht, in denen der Wahlausgang knapp war. Diese Stichprobe ist nicht unbedingt repräsentativ für die Grundgesamtheit aller Wahlkreise.

Warum sollte Gleichung 6 eine unverzerrte Schätzung des ATE ermöglichen, wo doch die Zuweisung zur Experimental- bzw. Kontrollgruppe im allgemeinen nicht zufällig erfolgt? Möglich wird dies durch die zumindest lokal randomisierte Zuweisung des Treatments in der unmittelbaren Nähe zum Schwellenwert $\bar{M} V_{i, j, t-1}$. Wie in Gleichungen 1 bis 3 dargestellt, ist der Stim-

10 Beweise hierfür bei Rubin (1977); Hahn et al. (2001); Lee (im Erscheinen).

11 Als weitere Voraussetzung für kausale Inferenz gehen wir davon aus, dass die SUTVABedingung erfüllt ist. SUTVA steht für „stable unit treatment variable assumption“ (Rubin 1978) und besagt, dass das Ergebnis für jede beliebige Einheit einzig von ihrer Zuweisung zur Experimental- oder Kontrollgruppe, nicht aber vom Treatment-Status anderer Einheiten abhängt. Die Tatsache, dass eine Partei der Amtsinhaber in einem Wahlkreis ist, darf also keinen Einfluss auf die potentiellen Ergebnisse dieser Partei in anderen Wahlkreisen haben. Diese Annahme ist nicht direkt überprüfbar. Sollte sie jedoch verletzt sein, werden kausale Schlüsse ungemein erschwert oder gar völlig unmöglich (Sobel 2003). Mit wenigen Ausnahmen setzen alle kausalen Inferenzen, inklusive derjenigen, die in der Forschung zu Kontaminationseffekten bislang vorgenommen wurden, diese Annahme voraus, wenngleich sie oft nicht explizit artikuliert wird. 
menabstand $M V_{t-1}$, also jene Hintergrundvariable, die über die Zuweisung zur Experimental- oder Kontrollgruppe entscheidet, eine Funktion des beobachteten Stimmenanteils $O V S_{i, j, t}$. Dieser wiederum setzt sich aus einer systematischen Komponente $\left(T V S_{i, j, t}\right)$, die von den Parteien beeinflusst werden kann, und einem vom Handeln der Parteien unabhängigen Zufallsterm $\left(\eta_{i, j}\right)$ zusammen. Solange aber die Hintergrundvariable, die über die Zuweisung zur Experimental- oder Kontrollgruppe entscheidet, eine solche Zufallskomponente mit stetiger Dichte beinhaltet, ist die Zuweisung des Treatments am Schwellenwert effektiv randomisiert.

Wenn die oben genannten Annahmen zutreffen, so sind in unmittelbarer Umgebung des Schwellenwertes alle beobachteten und unbeobachteten Störvariablen orthogonal zur Zuweisung des Treatments, wobei diese konditionale Unabhängigkeit formal ausgedrückt werden kann als: $Y_{i j}, Y_{0 i j} \perp D_{i, j, t} \mid M V_{i, j, t-1}=\bar{M} V_{i, j, t-1}$. Der kausale Effekt des Treatments kann somit wie in einem klassischen Zufallsexperiment unverzerrt geschätzt werden. Die Einbeziehung von (der Zuweisung des Treatments zeitlich vorausgehenden) Störvariablen sollte die Schätzung des kausalen Effekts substantiell kaum beeinflussen und sich lediglich in einer höheren Präzision der Schätzung niederschlagen. Die für eine unverzerrte Schätzung erforderliche konditionale Unabhängigkeitsbedingung ist im Falle beobachtbarer Störvariablen mit so genannten Balance-Tests überprüfbar, wie man sie von Zufallsexperimenten und MatchingVerfahren her kennt. Ist die lokale Randomisierung tatsächlich erfolgreich, dann sollte die Verteilung der Störvariablen in der Experimental- und Kontrollgruppe in der unmittelbaren Umgebung des Schwellenwertes gleich sein.

Wie bereits deutlich wurde, ist die Annahme lokaler Randomisierung in unmittelbarer Nähe zum Schwellenwert unverzichtbar für die Ermittlung des kausalen Effekts der Amtsinhaberschaft einer Partei auf ihren Erst- und Zweitstimmenanteil. Deshalb gilt es nach den Bedingungen zu fragen, unter denen diese zentrale Annahme nicht haltbar wäre. Lokale Randomisierung hängt entscheidend von der Existenz der oben angesprochenen Zufallskomponente $\left(\eta_{i, j, t}\right)$ ab. Diese Annahme impliziert jedoch nicht, dass in jedem Wahlkreis die Wahl von der Zufallskomponente entschieden werden muss - dies ist sicher meistens nicht der Fall. Maßgeblich ist nur, dass mit knapper werdendem Wahlausgang, also zunehmender Annäherung an den Schwellenwert, die Zuweisung des Treatments nicht mehr länger systematisch von verunreinigenden Störvariablen beeinflusst wird. In der Grenzbetrachtung sollte die Treatment-Zuweisung deshalb am kritischen Wert unabhängig von allen beobachtbaren wie auch unbeobachtbaren Störvariablen sein. Die Plausibilität dieser Annahme hängt davon ab, 
inwieweit Parteien sich in unmittelbarer Umgebung des kritischen Wertes gezielt positionieren können. Hätten Parteien beispielsweise vollständige Kontrolle über ihren beobachtbaren Stimmenanteil oder könnten sie diesen ohne Fehler vorhersagen, würden alle Parteien bis auf eine ihre Niederlage antizipieren und erst gar nicht antreten. Eine andere Möglichkeit wäre, dass Parteien lediglich genügend zusätzliche Anstrengungen unternähmen, um in einem Wahlkreis eine Stimme mehr als ihr stärkster Gegner zu erhalten. Ein solches Verhalten würde die Identifikationsannahme verletzen, da $E\left[Y_{0}\right]$ in unmittelbarer Umgebung des kritischen Wertes nicht mehr stetig wäre. Betrachtet man jedoch die Wählerverhalten innewohnende Zufälligkeit, erscheinen solche Szenarien nicht plausibel (Matsusaka und Palda 1999). Das Ausmaß von nicht steuerbaren, unsystematischen Zufallseinflüssen zeigt sich schon daran, dass das Wetter am Wahltag einen Einfluss auf die Wahlbeteiligung und das Wahlergebnis hat (Knack 1994).

Schließlich gilt es vor der empirischen Überprüfung noch eine mögliche Einschränkung unserer Analyse aufzuzeigen. Der hier ermittelte $A T E$ entspricht der Wirkung, welche die Amtsinhaberschaft einer Partei auf den Zweitstimmenanteil hat, wenn der Wahlausgang knapp ist. Solche Wahlkreise sind vermutlich nicht repräsentativ für die Grundgesamtheit aller Wahlkreise. Es gibt auch keinen Grund, davon auszugehen, dass sich Wahlkreise mit knappen und nicht knappen Wahlausgängen vollkommen gleichen. Sofern also keine zusätzlichen, mehr oder weniger willkürlichen Homogenitätsannahmen getroffen werden, beziehen sich die hier vorgestellten Schätzungen nur auf knappe Wahlkreise.

Knappe Wahlausgänge in den einzelnen Wahlkreisen treten jedoch erstaunlich häufig auf: Im Durchschnitt werden bei einer Bundestagswahl 20 Prozent aller Wahlkreise mit einem Vorsprung von weniger als 5 Prozentpunkten gewonnen. Letzten Endes ist die Begrenzung unserer Aussagen auf knappe Wahlkreise aber als das Resultat eines klassischen methodischen Zielkonfliktes zu begreifen. Auf der einen Seite erlaubt die RD-Analyse die Schätzung unverzerrter Kausaleffekte für die Amtsinhaberschaft einer Partei, aber eben nur für Wahlkreise mit knappem Wahlausgang. Auf der anderen Seite wäre es wünschenswert, kausale Effekte für die Grundgesamtheit aller Wahlkreise zu schätzen. Hierfür wären jedoch zusätzlich starke Annahmen über jene Mechanismen erforderlich, die über die Zuweisung des Treatments entscheiden. So müsste man beispielsweise annehmen, dass der Amtsinhaberstatus in allen Wahlkreisen (nachdem für eine oder mehrere Störvariablen kontrolliert wurde) nach dem Zufallsprinzip verliehen wird. Zudem müssten Parteien diejenigen Wahlkreise, in denen sie mit einem eigenen Kandidaten antreten, rein zufällig auswählen. Falls diese Annahmen zuträfen, könnte die Zuweisung des Treatments ignoriert 
werden (ignorable treatment assignment) und somit der kausale Effekt der Amtsinhaberschaft für alle Wahlkreise unverzerrt geschätzt werden. Unserer Meinung nach entbehren solche Annahmen jeglicher empirischer Grundlage. Deshalb entscheiden wir an dieser Stelle mit der RD-Analyse zu Gunsten der internen Validität (Shadish et al. 2002).

\section{Empirische Schätzung}

Das entwickelte Modell legt den folgenden Datengenerierungsprozess für die beobachteten Stimmenanteile nahe:

$Y_{i, j, t}=f\left(Z_{i, j, t-1}, \theta\right)+h\left(M V_{i, j, t-1}, \delta\right)+D_{i, j} \beta+\varepsilon_{i, j}$

Funktion $f$ gibt an, wie eine Matrix wahlkreisspezifischer Kovariaten $Z_{i, j, t-1}$ (zum Beispiel Kandidatenpersönlichkeit, Wahlkampfanstrengungen) den Stimmenanteil mit dem Koeffizientenvektor $\theta$ beeinflusst. $h$ ist eine Funktion, die den Stimmenabstand bei der vorherigen Wahl in jedem Wahlkreis mit dem Stimmenanteil in der nächsten Wahl in Verbindung bringt. $D$ ist der TreatmentIndikator und $\beta$ somit der Parameter, welcher den kausalen Effekt der Amtsinhaberschaft einer Partei misst.

Auf den ersten Blick erscheint Gleichung 7 als konventionelles Regressionsmodell, wie es in der Kontaminationsforschung bislang Verwendung gefunden hat. Die Interpretation früherer Ergebnisse ist jedoch problematisch, da ein $Z$ existieren könnte, das nicht beobachtbar ist und für das in einer StandardRegressionsanalyse folglich auch nicht kontrolliert werden kann. Da $Z$ sehr wahrscheinlich mit $M V$ korreliert, sind die unter diesen Bedingungen ermittelten Schätzungen verzerrt. Im Gegensatz dazu stellt die Präsenz von solchen unbeobachteten Störvariablen für die RD-Analyse kein Problem dar. Treffen die oben beschriebenen Annahmen zu, dann wird die lokal randomisierte TreatmentZuweisung dafür sorgen, dass die Schätzung für $\beta$ in unmittelbarer Umgebung des kritischen Wertes $M V_{i, j, t-1}=\bar{M} V_{i, j, t-1}=0$ unverzerrt ist. Da über die Zuwei-

sung des Treatments in der Nähe des Schwellenwertes zufällig entschieden wird, ist keine Kontrolle für Störvariablen nötig. Genau wie in einem echten Zufallsexperiment sollte der Einschluss zusätzlicher Störvariablen die Schätzung des kausalen Effektes nicht stark beeinflussen, sondern lediglich die Präzision der Schätzung erhöhen. 
In RD-Analysen ist die Wahl der richtigen funktionalen Form für $h$ von zentraler Bedeutung. Das Ziel besteht darin, $D$ nur den potentiellen Sprung der bedingten Erwartung $E\left[Y \mid M V_{i, j, t-1}\right]$ in unmittelbarer Nähe des Schwellenwertes erfassen zu lassen. Die gängige Lösung (Lee (im Erscheinen); Butler und Butler 2005) besteht in der Wahl einer möglichst flexiblen funktionalen Form für $E\left[Y \mid M V_{i, j, t-1}\right]$, indem von $M V_{i, j, t-1}$ ein Polynom vierten Grades sowie alle Interaktionsterme mit dem Treatment-Indikator aufgenommen werden. ${ }^{12} \mathrm{Wir}$ folgen dieser Strategie und verwenden ebenfalls ein multiplikatives Polynom vierten Grades, wobei sich unsere Ergebnisse nicht verändern, wenn stattdessen ein Polynom dritten oder fünften Grades verwendet wird. ${ }^{13} \mathrm{Um}$ die Wahrscheinlichkeit einer Fehlspezifikation weiter zu verringern, wird zudem ein MMSchätzer verwendet. Damit vermeiden wir die allgemein bekannte Anfälligkeit des KQ-Schätzers für Ausreißer (Yohai 1987, Yohai et al. 1991). Der MMSchätzer bietet den Vorteil hoher Robustheit bei hoher Effizienz. ${ }^{14}$ Die stabile, asymptotische Formel von Croux (2003) liefert zudem robuste, also gegen Heteroskedastie und serielle Korrelation widerstandsfähige Standardfehler.

Unsere Daten stammen von Caramani (2000). War ursprünglich noch beabsichtigt, alle deutschen Bundestagswahlen $\mathrm{zu}$ analysieren, musste diese Absicht auf Grund mehrerer Neueinteilungen der Wahlkreise aufgegeben werden. Deshalb sind die Bundestagswahlen 1957, 1976, 1980 und 2002 von der Untersuchung ausgeschlossen. Letztlich verbleiben neun Bundestagswahlen mit 248

12 Geschätzt wird also die folgende Gleichung:

$Y=\delta_{0}+\delta_{1} M V+\delta_{2} M V^{2}+\delta_{3} M V^{3}+\delta_{4} M V^{4}+\beta D+\delta_{5}(D \times M V)+\delta_{6}\left(D \times M V^{2}\right)$ $+\delta_{7}\left(D \times M V^{3}\right)+\delta_{8}\left(D \times M V^{4}\right)$.

Der Koeffizient für $D$ identifiziert den ATE, da der kritische Wert der Zuweisungsvariablen $M V$ bei Null liegt.

13 Diese Ergebnisse sind auf Anfrage erhältlich.

14 Unsere Implementierung des MM-Schätzers erfolgt nach der dreistufigen Methode von Yohai (1987). Zunächst wird mit Hilfe eines S-Schätzers eine vorläufige, hochrobuste Schätzung entsprechend Rousseeuw und Yohai (1984) ermittelt. Im zweiten Schritt wird eine M-Schätzung der Fehlerskala mittels der Residuen der ersten Schätzung vorgenommen. Schließlich können mit einem M-Schätzer die Regressionskoeffizienten mittels der Methode iterativer, umgewichteter kleinster quadrierter Abweichungen (iteratively reweighted least squares (IRLS)) berechnet werden. Der so implementierte MM-Schätzer ist hochrobust und in der Lage, die korrekten Parameter selbst dann zu identifizieren, wenn der Datensatz bis zur Hälfte verunreinigt ist. Dies steht im starken Gegensatz zur Anfälligkeit des KQ-Schätzers für Ausreißer, bei dem die Verschiebung eines einzigen Datenpunktes ausreicht, um die Hyperebene in jede beliebige Richtung zu drehen. 
Wahlkreisen vor und 328 nach der Wiedervereinigung. ${ }^{15}$ In nahezu allen Wahlkreisen sind SPD und CDU/CSU die beiden stärksten Parteien. Vor diesem Hintergrund konzentrieren wir uns im Folgenden auf die Wirkung der Amtsinhaberschaft dieser beiden Parteien auf ihren Erst- und Zweitstimmenanteil. ${ }^{16}$

Zunächst wurden die Stimmenabstände für SPD und CDU/CSU entsprechend den Gleichungen 2 und 3 berechnet. Die Wirkung des Amtsinhaberstatus einer Partei wurde sodann durch die in Gleichung 7 dargestellte Spezifikation geschätzt. Der kausale Effekt des Amtsinhaberstatus ist dabei der Sprung des konditionalen Erwartungswertes $Y$ auf Höhe des Schwellenwerts $\bar{M} V$. Es werden somit für jede der beiden Parteien knapp gewonnene Wahlkreise mit knapp verlorenen Wahlkreisen kontrastiert.

\section{Ergebnisse}

Wie aus der oberen Hälfte von Tabelle 1 ersichtlich, wirkt sich der Amtsinhaberstatus sowohl für die SPD als auch für die CDU/CSU positiv auf den Erststimmenanteil aus. Hinsichtlich Größe und Signifikanz sind die Effekte für SPD und CDU/CSU sehr ähnlich. Im Durchschnitt erhöht der Amtsinhaberstatus einer Partei den Erststimmenanteil ihres Kandidaten um etwa 1,5 Prozentpunkte. Die entsprechenden Koeffizienten sind mindestens auf dem 10\%-Niveau signifikant. Diese Ergebnisse sind weitgehend mit den in vorherigen Studien erzielten vergleichbar (Bawn 1999; Moser und Scheiner 2005). ${ }^{17}$

15 Da jede Neueinteilung der Bundestagswahlkreise die Ergebnisse von zwei Bundestagswahlen für unsere Analyse unbrauchbar macht (da wir auch die Stimmenanteile der vorhergehenden Bundestagswahl benötigen), beinhaltet unser Datensatz die Bundestagswahlen der Jahre 1961, 1969, 1972, 1983, 1987, 1990, 1994, und 1998. In der Wahlforschung wird regelmäßig auf die Unterschiede im Wahlverhalten zwischen Ost- und Westdeutschland hingewiesen (Jeffery und Hough 2003; Hough und Jeffery 2001; Falter et al. 2000; siehe jedoch Kern und Hainmüller 2006). Unsere Ergebnisse ändern sich nicht, wenn die Wahlkreise in den neuen Bundesländern von der Analyse ausgenommen werden. Die Resultate sind auf Anfrage erhältlich.

16 Auch kleine Parteien wie Grüne und FDP treten in nahezu jedem Wahlkreis an. Schließt man die wenigen Wahlkreise aus, in denen dies nicht der Fall ist, ändern sich unsere Ergebnisse nicht.

17 Wenngleich zu betonen ist, dass vorherige Arbeiten lediglich den Vorteil von sich erneut zur Wahl stellenden Wahlkreisabgeordneten ermittelt haben. Wir hingegen fragen, inwieweit sich die Amtsinhaberschaft einer Partei, das heißt die Tatsache, dass ihr Kandidat bei der vergangenen Wahl den größten Stimmenanteil im Wahlkreis erringen konnte, auf ihren Stimmenanteil in der nächsten Wahl auswirkt. Ob der amtierende Wahlkreisabgeordnete selbst wieder zur Wahl steht oder nicht bleibt dabei unbeachtet. 
Tabelle 1: Der kausale Effekt des Amtsinhaberstatus einer Partei auf den Erstund Zweitstimmenanteil

Effekt auf Erststimmenanteil

\begin{tabular}{lccccc}
\hline & Amtsinhaber & $\begin{array}{c}\text { Nicht- } \\
\text { Amtsinhaber }\end{array}$ & ATE & $\begin{array}{c}\text { Untere Schranke } \\
90 \%-K I\end{array}$ & $\begin{array}{c}\text { Obere Schranke } \\
90 \%-K I\end{array}$ \\
\hline SPD & 44,598 & 46,197 & 1,6 & 0,325 & 2,874 \\
& $(0,440)$ & $(0,637)$ & $(0,775)$ & & \\
CDU & 41,718 & 43,091 & 1,373 & 0,199 & 2,548 \\
& $(0,527)$ & $(0,481)$ & $(0,714)$ & & \\
\hline
\end{tabular}

Effekt auf Zweitstimmenanteil

\begin{tabular}{lccrcc}
\hline & Amtsinhaber & $\begin{array}{c}\text { Nicht- } \\
\text { Amtsinhaber }\end{array}$ & ATE & $\begin{array}{c}\text { Untere Schranke } \\
90 \% \text {-KI }\end{array}$ & $\begin{array}{c}\text { Obere Schranke } \\
90 \% \text {-KI }\end{array}$ \\
\hline SPD & 41,885 & 43,376 & 1,491 & 0,160 & 2,823 \\
& $(0,488)$ & $(0,646)$ & $(0,809)$ & & \\
CDU & 38,998 & 40,055 & 1,057 & $-0,225$ & 2,339 \\
& $(0,590)$ & $(0,510)$ & $(0,779)$ & & \\
\hline
\end{tabular}

MM-Schätzungen mit robusten Standardfehlern in Klammern. Alle Schätzungen basieren auf einem multiplikativen Polynom vierten Grades gebildet aus der Zuweisungsvariable und dem Treatment-Indikator links und rechts des Schwellenwertes. Gezeigt wird nur der average treatment effect (ATE). Jede Zeile stellt eine separate Schätzung dar. $\mathrm{KI}=$ Konfidenzintervall. $\mathrm{N}=1972$.

Die untere Hälfte von Tabelle 1 zeigt die Schätzungen für den Effekt des Amtsinhaberstatus auf den Zweitstimmenanteil. Hier wird deutlich, dass sich die Amtsinhaberschaft einer Partei positiv auf den Zweitstimmenanteil auswirkt, wobei der Effekt bei 1,5 Prozentpunkten für die SPD und knapp über einem Prozentpunkt für die CDU liegt. ${ }^{18}$ Die Schätzung für die SPD ist auf dem $10 \%$ Niveau signifikant, für die CDU/CSU werden konventionelle Signifikanzniveaus knapp verfehlt. ${ }^{19}$

18 Die Konfidenzintervalle der beiden Schätzungen überschneiden sich weitgehend, deshalb ist der Unterschied in den Punktschätzungen für die beiden Parteien mit Vorsicht zu interpretieren.

19 Hier wird die geringe Teststärke als ein Nachteil der RD-Analyse deutlich, das heisst die starke Neigung, die Alternativhypothese fälschlicherweise abzulehnen (Typ II-Fehler). Wie jedoch noch gezeigt werden wird, können diese Schätzungen durch die Hinzunahme von möglichen Störvariablen präzisiert werden. 
Inwieweit lässt sich der durch die Parteizugehörigkeit des Amtsinhabers verursachte Kontaminationseffekt mit den Ergebnissen früherer Arbeiten zur Kontamination in kombinierten Wahlsystemen vergleichen? Wie oben bereits dargestellt, haben sich bisherige Arbeiten vornehmlich auf den Kontaminationseffekt konzentriert, der von der bloßen Aufstellung eines Wahlkreisbewerbers ausgeht. Ferrara et al. (2005: 44) berichten von solchen Kontaminationseffekten im Fall mehrerer kleiner (ungenannter) Parteien bei der Bundestagswahl 1953, präsentieren aber keine Punktschätzungen. Cox und Schoppa (2002) können nicht ermitteln, wie sich die Aufstellung eines eigenen Wahlkreiskandidaten in Bundestagswahlen auswirkt, da ihre unabhängige Variable keine Varianz aufweist, das heißt alle kleinen Parteien mit eigenen Kandidaten in fast jedem Wahlkreis antreten. Ferrara et al. (2005) können zumindest feststellen, dass in Russland, Japan und einigen anderen Ländern das Aufstellen eines eigenen Wahlkreiskandidaten eine positive Wirkung auf den Stimmenanteil der Parteien hat. Ihre Punktschätzungen schwanken in Abhängigkeit von der betrachteten Partei zwischen 0 und 5 Prozentpunkten. Für japanische Parteien ermitteln Herron und Nishkawa (2002) eine noch stärkere Kontaminationswirkung. Im Vergleich dazu sind unsere Schätzungen der durch den Amtsinhaberstatus verursachten Kontamination von geringerer Größe. Es ist natürlich durchaus möglich, dass der Amtsinhaberstatus einer Partei eine weniger bedeutende Ursache von Kontamination ist als die Gegenwart eines eigenen Wahlkreisbewerbers. Obgleich diese Frage hier nicht abschließend beantwortet werden kann, halten wir es aber durchaus für möglich, dass frühere Befunde durch Selbstauswahl verzerrt wurden. Wenn Parteien bei der Kandidatenaufstellung tatsächlich strategisch agieren und Kandidaten vor allem in denjenigen Wahlkreisen aufstellen, in denen sie sich ein besseres Wahlergebnis erhoffen, wären frühere Schätzungen nach oben hin verzerrt.

Abschließend stellt Abbildung 1 unsere Ergebnisse für den kausalen Effekt der Amtsinhaberschaft auf den Erst- und Zweitstimmenanteil graphisch dar. Die Ordinate zeigt den Stimmenanteil einer Partei im Wahlkreis zum Zeitpunkt $t$. Auf der Abszisse ist der Stimmenabstand im selben Wahlkreis zum Zeitpunkt $t-1$ abgetragen, wobei die gestrichelte vertikale Linie den Schwellenwert markiert. Alle in der Experimentalgruppe (Kontrollgruppe) befindlichen Beobachtungen liegen rechts (links) des Schwellenwertes. Links und rechts des Schwellenwertes verläuft die geschätzte polynomische Funktion. Jedes Element der Punktewolke steht für einen lokalen Durchschnitt der Ergebnisvariablen (Erststimmen- bzw. Zweitstimmenanteil), der für jedes 0,05 breite Intervall der Abszisse berechnet wurde. 
Abbildung 1: Der kausale Effekt des Amtsinhaberstatus einer Partei auf den Erst- und Zweitstimmenanteil

SPD: Effekt auf Erststimmenanteil

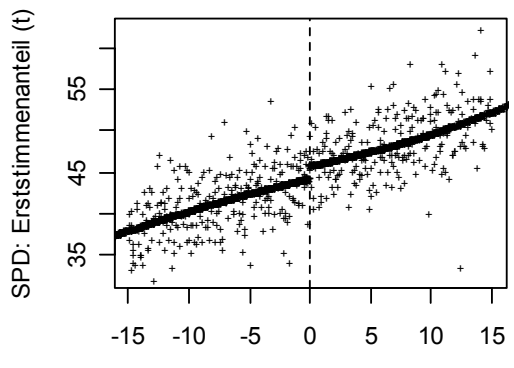

SPD: Erststimmenabstand (t-1)

SPD: Effekt auf Zweitstimmenanteil

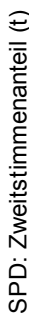

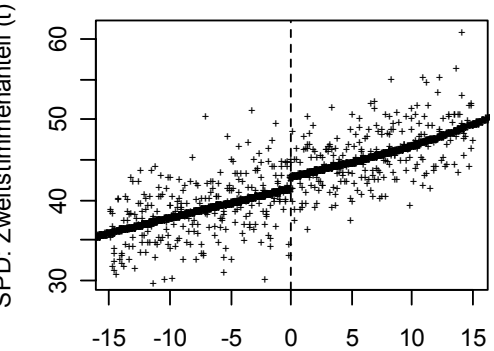

SPD: Erststimmenabstand (t-1)
CDU: Effekt auf Erststimmenanteil

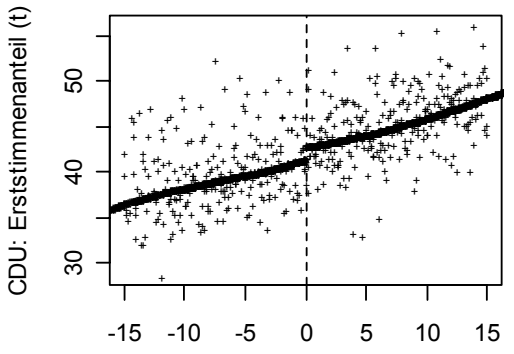

CDU: Erststimmenabstand (t-1)

CDU: Effekt auf Zweitstimmenanteil

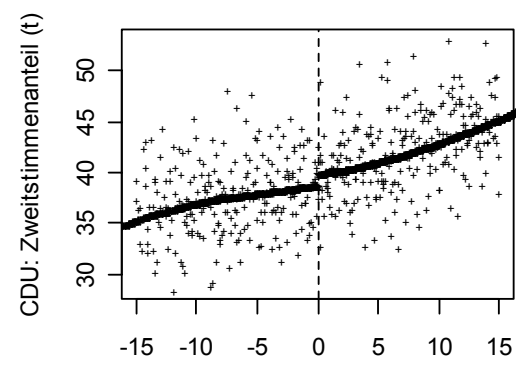

CDU: Erststimmenabstand (t-1)

Auffällig ist für beide Parteien der positive Zusammenhang zwischen Stimmenabstand und Stimmenanteil. Gewinnt (verliert) eine Partei mit einem großen Stimmenabstand zum Zeitpunkt $t-1$, wird sie bei der nächsten Wahl $t$ meist wieder einen hohen (niedrigen) Stimmenanteil erhalten. Noch bedeutender als dieser positive Zusammenhang ist allerdings der deutlich erkennbare Sprung an jedem der vier Schwellenwerte, der den durchschnittlichen kausalen Effekt (ATE) des Amtsinhaberstatus einer Partei repräsentiert. Hätte Amtsinhaberschaft keine kausale Wirkung, gäbe es keine solchen Sprungstellen. Abgesehen vom Schwellenwert existieren keine weiteren Unstetigkeiten in den konditionalen Erwartungsfunktionen. Der empirische Zusammenhang zwischen dem 
Stimmenabstand und den Ergebnisvariablen wird durch unsere multiplikative polynomische Funktion in allen vier Fällen gut angenähert.

Wie ist die Größenordnung der hier geschätzten Kontaminationseffekte zu interpretieren? Ist die durch den Amtsinhaberstatus verursachte Kontamination groß genug, um signifikante Veränderungen in den Mehrheitsverhältnissen des Bundestages auszulösen? Um hierauf eine Antwort geben zu können, wurde die Verteilung der Bundestagsmandate mit und ohne Kontaminationseffekt numerisch simuliert. ${ }^{20}$ Unsere Simulationen basieren dabei auf den in Tabelle 1 dargestellten Ergebnissen. Demnach führt die durch den Amtsinhaberstatus ausgelöste Kontamination im Durchschnitt zu Nettoverschiebungen von drei bis neun Bundestagsmandaten. Kleinere Parteien würden ohne Kontamination besser abschneiden, da sie so gut wie nie Wahlkreisabgeordnete stellen. Die SPD profitiert etwas mehr von der kontaminierenden Wirkung des Amtsinhaberbonus als die CDU/CSU.

Um die Relevanz des Kontaminationseffekts zu verdeutlichen, gehen wir an dieser Stelle näher auf die Simulationsergebnisse für die Bundestagswahl 2002 ein. In dieser Wahl standen sich Bundeskanzler Gerhard Schröder, gestützt von SPD und Grünen, und sein Herausforderer Edmund Stoiber, der Kanzlerkandidat einer möglichen Koalition aus CDU/CSU und FDP, gegenüber. Das

20 In einem ersten Schritt wurde eine Funktion (mittels R) programmiert, die von den gültigen Erst- und Zweitstimmenanteilen für alle Parteien in allen Wahlkreisen ausgeht. Diese Funktion berechnet auf dieser Basis die Sitzverteilung im Bundestag unter Berücksichtigung der vom deutschen Wahlgesetz vorgeschriebenen Umrechnungsregeln. Auch Überhangmandate finden dabei Berücksichtigung. In einem zweiten Schritt wurde eine weitere Funktion programmiert, mit der die Sitzverteilung ohne Kontaminationseffekte simuliert wird. Diese Funktion verwendet die ursprünglichen Erststimmenanteile aller Parteien zum Zeitpunkt $t$-1, um den Amtsinhaber des jeweiligen Wahlkreises zu bestimmen. Daran anschließend werden die Zweitstimmenanteile zum Zeitpunkt $t$ in jedem Wahlkreis neu verteilt. Eine Partei, deren Kandidat in einem Wahlkreis das Direktmandat innehat, verliert Zweitstimmen in Höhe des geschätzten Kontaminationseffekts. Diese Stimmen werden dann proportional auf alle im gleichen Wahlkreis angetretenen Parteien verteilt. Damit erhalten wir eine aus kontrafaktischen Zweitstimmenanteilen bestehende Matrix. Diese Matrix gibt für jeden Wahlkreis und jede Partei jenen Anteil an Zweitstimmen zum Zeitpunkt $t$ wider, der in einer kontrafaktischen Welt ohne Kontamination durch die Amtsinhaberschaft einer Partei zu beobachten gewesen wäre. Unsere erste Funktion nutzt diese Matrix, um die daraus resultierenden Änderungen in der Sitzverteilung des Bundestags zu ermitteln. Jede Simulation wurde zweimal durchgeführt, um der Unsicherheit in unseren Schätzungen Rechnung zu tragen (siehe Tabelle 1). Die erste Simulation beruht auf der unteren Schranke des Konfidenzintervalls, die zweite auf der oberen. Hierbei ist anzumerken, dass unsere Simulationen nur die kontaminierende Wirkung der Amtsinhaberschaft einer Partei in Rechnung stellen. Andere Ursachen von Kontamination wie die Existenz von Wahlkreiskandidaten werden somit konstant gehalten. 
tatsächliche Wahlergebnis ist in der ersten Zeile von Tabelle 2 zu finden. Mit einer knappen Mehrheit von 306 der insgesamt 603 Sitze gewann die rot-grüne Koalition die erforderliche Kanzlermehrheit. CDU/CSU und FDP verpassten mit 295 Mandaten die Mehrheit um sieben Sitze. Die zweite und dritte Spalte der Tabelle 2 zeigen die Sitzverteilung in einer kontrafaktischen Bundestagswahl 2002 ohne Kontamination.

CDU/CSU und die FDP hätten in diesem Fall möglicherweise die Mehrheit der Bundestagsmandate errungen. Mit der erforderlichen Kanzlermehrheit wäre dann Edmund Stoiber zum Regierungschef gewählt worden. Deutlich wird auch, dass kleine Parteien besonders stark unter dem Kontaminationseffekt leiden. Hätte die Amtsinhaberschaft in der Bundestagswahl 2002 keine Wirkung auf den Zweitstimmenanteil gehabt, hätte die FDP vier bis elf zusätzliche Sitze erwerben können; ihre Bundestagsfraktion wäre demnach statt der tatsächlichen 47 zwischen 51 und 58 Mitglieder stark gewesen. Auch die Grünen hätten es ohne Kontamination leichter gehabt, wären sie doch in den Genuss von zwei bis fünf zusätzlichen Bundestagsmandaten gekommen. Lediglich für die PDS ergibt sich bei der Bundestagswahl 2002 keine negative Wirkung auf ihren Zweitstimmenanteil, da sie weder drei Direktmandate erringen noch die 5\%-Hürde überwinden konnte.

Tabelle 2: Sitzverteilung des 15. Deutschen Bundestags mit und ohne Kontaminationseffekt (Bundestagswahl 2002)

\begin{tabular}{|c|c|c|c|}
\hline \multirow[t]{2}{*}{ Partei } & \multirow[t]{2}{*}{$\begin{array}{l}\text { Mit Kontamination } \\
\text { (tatsächliche Sitzverteilung) }\end{array}$} & \multicolumn{2}{|c|}{$\begin{array}{l}\text { Ohne Kontamination } \\
\text { (kontrafaktische Sitzverteilung) }\end{array}$} \\
\hline & & $\begin{array}{l}\text { Untere Schranke } \\
90 \% \mathrm{KI}\end{array}$ & $\begin{array}{l}\text { Obere Schranke } \\
90 \% \mathrm{KI}\end{array}$ \\
\hline $\mathrm{CDU} / \mathrm{CSU}$ & 248 & 247 & 246 \\
\hline FDP & 47 & 51 & 58 \\
\hline SPD & 251 & 248 & 243 \\
\hline Grüne & 55 & 57 & 60 \\
\hline PDS & 2 & 2 & 2 \\
\hline Total & 603 & 605 & 609 \\
\hline Mehrheit & 302 & 303 & 305 \\
\hline CDU/FDP & 295 & 298 & 304 \\
\hline SPD/Grüne & 306 & 305 & 303 \\
\hline
\end{tabular}

Die Simulation basiert auf den in Tabelle 1 dargestellten Ergebnissen. KI = Konfidenzintervall. 
Die hier präsentierten Schätzungen basieren auf der Annahme randomisierter Treatment-Zuweisung in der Nähe des Schwellenwertes (lokale Randomisierung). In der folgenden Sensitivitätsanalyse wenden wir uns nun der Frage zu, inwieweit diese zentrale Annahme gerechtfertigt erscheint.

\section{Sensitivitätsanalyse}

In einem randomisierten Experiment, in welchem die Einheiten nach dem $\mathrm{Zu}$ fallsprinzip der Experimental- oder Kontrollgruppe zugeordnet werden, gleichen sich beide Gruppen - zumindest in der Grenzbetrachtung - in Bezug auf die Verteilung aller beobachteten und unbeobachteten Störvariablen. Die Gruppen sind deshalb austauschbar und jeder Unterschied zwischen den Gruppen nach der Treatment-Zuweisung lässt sich kausal auf den Effekt des Treatments zurückführen. $\mathrm{Ob}$ die Randomisierung tatsächlich erfolgreich war, lässt sich zumindest hinsichtlich der Verteilung beobachteter Störfaktoren mittels so genannter Balance-Tests leicht überprüfen. Falls in einer RD-Analyse die Annahme randomisierter Treatment-Zuweisung nahe dem Schwellenwert zutreffend ist, sollten sich beide Gruppen in unmittelbarer Umgebung dieses kritischen Wertes ebenfalls annähernd gleichen. Beispielsweise dürfte es keine systematischen Unterschiede zwischen der Wahlbeteiligung zum Zeitpunkt $t$ - 1 in knapp verlorenen und knapp gewonnen Wahlkreisen geben. Gleiches gilt für quadrierte Variablen, Interaktionsterme und andere lineare Kombinationen der Störvariablen. Die Tabellen 3 und 4 zeigen die Ergebnisse solcher Balance-Tests für beobachtete potentielle Störfaktoren.

In der Nähe des Schwellenwertes bestehen weder für die SPD noch für die CDU/CSU hinsichtlich Wahlbeteiligung, Erst- und Zweitstimmenanteil sowie linearen Kombinationen dieser Variablen systematische Unterschiede. Diese Resultate lassen somit die Annahme lokaler Randomisierung gerechtfertigt erscheinen. Natürlich lassen sich auf diese Weise nur die Verteilungen beobachteter Störfaktoren überprüfen. Der zentrale Vorteil von randomisierten Experimenten wie auch der RD-Analyse liegt jedoch darin, dass man plausiblerweise davon ausgehen kann, dass die lokale Randomisierung die Gruppen nicht nur in Bezug auf alle beobachteten, sondern auch in Bezug auf alle unbeobachteten Störfaktoren ausgeglichen hat. Neben der Durchführung von Balance-Tests können unsere Befunde durch eine zweite Art von Sensitivitätsanalyse auf den Prüfstand gestellt werden. Wie in einem Experiment, in dem die TreatmentZuweisung erfolgreich randomisiert wurde, sollten sich unsere Schätzungen 
nicht stark verändern, wenn dem statistischen Modell weitere, der TreatmentZuweisung zeitlich vorausgehende Störvariablen hinzugefügt werden. Lediglich die Präzision, mit welcher die Treatment-Effekte geschätzt werden können, sollte sich erhöhen, falls die hinzugefügten Störvariablen selbst eine gewisse Vorhersagekraft in Bezug auf die Ergebnisvariable aufweisen. In Tabelle 5 und Tabelle 6 wird ersichtlich, dass dies in der Tat der Fall ist.

Tabelle 3: Überprüfung der lokalen Randomisierung anhand von BalanceTests für Störvariablen: SPD

\begin{tabular}{cccccc}
\hline & $\begin{array}{c}\text { Amts- } \\
\text { inhaber }\end{array}$ & $\begin{array}{c}\text { Nicht- } \\
\text { Amts- } \\
\text { inhaber }\end{array}$ & $\begin{array}{c}\text { Diff. am } \\
\text { Schwel- } \\
\text { lenwert }\end{array}$ & $\begin{array}{c}\text { Untere } \\
\text { Schranke } \\
90 \%-K I\end{array}$ & $\begin{array}{c}\text { Obere } \\
\text { Schranke } \\
90 \%-K I\end{array}$ \\
\cline { 2 - 6 } Erststimmen- & 44,470 & 44,131 & $-0,339$ & $-0,945$ & 0,266 \\
anteil (t-1) & $(0,230)$ & $(0,291)$ & $(0,368)$ & & \\
Erststimmen- & 1981,280 & 1954,897 & $-26,382$ & $-80,047$ & 27,282 \\
anteil^2 (t-1) & $(19,249)$ & $(26,534)$ & $(32,626)$ & & \\
Zweitstimmen- & 42,079 & 42,076 & $-0,003$ & $-0,731$ & 0,725 \\
anteil (t-1) & $(0,273)$ & $(0,350)$ & $(0,443)$ & & \\
Zweitstimmen- & 1776,106 & 1774,233 & $-1,873$ & $-63,492$ & 59,747 \\
anteil^2 (t-1) & $(21,549)$ & $(30,649)$ & $(37,462)$ & & \\
Wahlbeteiligung (t) & 85,712 & 85,078 & $-0,635$ & $-1,895$ & 0,625 \\
& $(0,441)$ & $(0,630)$ & $(0,766)$ & & \\
Wahlbeteiligung^2 (t) & 7345,706 & 7243,136 & $-102,571$ & $-317,093$ & 111,951 \\
& $(75,443)$ & $(106,827)$ & $(130,420)$ & & \\
Erststimmenanteil $\times$ & 3791,533 & 3734,092 & $-57,441$ & $-154,581$ & 39,698 \\
Wahlbeteiligung & $(35,236)$ & $(47,427)$ & $(59,057)$ & & \\
Zweitstimmenanteil $\times$ & 3791,533 & 3734,092 & $-57,441$ & $-154,581$ & 39,698 \\
Wahlbeteiligung & $(35,236)$ & $(47,427)$ & $(59,057)$ & & \\
Erststimmenanteil $\times$ & 1871,528 & 1860,201 & $-11,328$ & $-66,887$ & 44,232 \\
Zweitstimmenanteil & $(19,858)$ & $(27,364)$ & $(33,778)$ & & \\
& & & & &
\end{tabular}

MM-Schätzungen mit robusten Standardfehlern in Klammern. Alle Schätzungen basieren auf einem multiplikativen Polynom vierten Grades gebildet aus der Zuweisungsvariable und dem Treatment-Indikator links und rechts des Schwellenwertes. Gezeigt wird nur der jeweilige ATE (average treatment effect). Jede Zeile stellt eine separate Schätzung dar. $\mathrm{KI}=$ Konfidenzintervall. $\mathrm{N}=197$. 
Tabelle 4: Überprüfungen der lokalen Randomisierung anhand von BalanceTests für Störvariablen: CDU

\begin{tabular}{|c|c|c|c|c|c|}
\hline & $\begin{array}{l}\text { Amts- } \\
\text { inhaber }\end{array}$ & $\begin{array}{l}\text { Nicht- } \\
\text { Amts- } \\
\text { inhaber }\end{array}$ & $\begin{array}{c}\text { Diff. am } \\
\text { Schwellen- } \\
\text { wert }\end{array}$ & $\begin{array}{c}\text { Untere } \\
\text { Schranke } \\
90 \% \text {-KI }\end{array}$ & $\begin{array}{c}\text { Obere } \\
\text { Schranke } \\
90 \%-\mathrm{KI}\end{array}$ \\
\hline $\begin{array}{l}\text { Erststimmen- } \\
\text { anteil (t-1) }\end{array}$ & $\begin{array}{l}44,130 \\
(0,291)\end{array}$ & $\begin{array}{l}44,506 \\
(0,230)\end{array}$ & $\begin{array}{c}0,376 \\
(0,368)\end{array}$ & $-0,230$ & 0,982 \\
\hline $\begin{array}{l}\text { Erststimmen- } \\
\text { antei1^2 }(\mathrm{t}-1)\end{array}$ & $\begin{array}{r}1943,999 \\
(24,987)\end{array}$ & $\begin{array}{r}1979,665 \\
(21,872)\end{array}$ & $\begin{array}{c}35,666 \\
(33,029)\end{array}$ & $-18,661$ & 89,993 \\
\hline $\begin{array}{l}\text { Zweitstimmen- } \\
\text { anteil (t-1) }\end{array}$ & $\begin{array}{l}41,723 \\
(0,397)\end{array}$ & $\begin{array}{l}41,786 \\
(0,296)\end{array}$ & $\begin{array}{c}0,062 \\
(0,495)\end{array}$ & $-0,752$ & 0,877 \\
\hline $\begin{array}{l}\text { Zweitstimmen- } \\
\text { anteil`2 (t-1) }\end{array}$ & $\begin{array}{r}1743,195 \\
(31,882)\end{array}$ & $\begin{array}{r}1753,653 \\
(26,262)\end{array}$ & $\begin{array}{c}10,458 \\
(41,295)\end{array}$ & $-57,466$ & 78,383 \\
\hline Wahlbeteiligung (t) & $\begin{array}{l}85,087 \\
(0,629)\end{array}$ & $\begin{array}{l}85,779 \\
(0,442)\end{array}$ & $\begin{array}{c}0,693 \\
(0,766)\end{array}$ & $-0,567$ & 1,952 \\
\hline Wahlbeteiligung ${ }^{\wedge} 2(\mathrm{t})$ & $\begin{array}{l}7244,716 \\
(106,685)\end{array}$ & $\begin{array}{r}7357,171 \\
(75,689)\end{array}$ & $\begin{array}{c}112,456 \\
(130,432)\end{array}$ & $-102,085$ & 326,996 \\
\hline $\begin{array}{l}\text { Erststimmenanteil } \times \\
\text { Wahlbeteiligung }\end{array}$ & $\begin{array}{r}3734,197 \\
(45,500)\end{array}$ & $\begin{array}{r}3791,866 \\
(37,338)\end{array}$ & $\begin{array}{c}57,669 \\
(58,768)\end{array}$ & $-38,995$ & 154,333 \\
\hline $\begin{array}{l}\text { Zweitstimmenanteil } \times \\
\text { Wahlbeteiligung }\end{array}$ & $\begin{array}{r}3734,197 \\
(45,500)\end{array}$ & $\begin{array}{r}3791,866 \\
(37,338)\end{array}$ & $\begin{array}{c}57,669 \\
(58,768)\end{array}$ & $-38,995$ & 154,333 \\
\hline $\begin{array}{l}\text { Erststimmenanteil } \times \\
\text { Zweitstimmenanteil }\end{array}$ & $\begin{array}{r}1838,505 \\
(27,704)\end{array}$ & $\begin{array}{r}1858,710 \\
(23,178)\end{array}$ & $\begin{array}{c}20,205 \\
(36,067)\end{array}$ & $-39,119$ & 79,530 \\
\hline
\end{tabular}

MM-Schätzungen mit robusten Standardfehlern in Klammern. Alle Schätzungen basieren auf einem multiplikativen Polynom vierten Grades gebildet aus der Zuweisungsvariable und dem Treatment-Indikator links und rechts des Schwellenwertes. Gezeigt wird nur der jeweilige ATE (average treatment effect). Jede Zeile stellt eine separate Schätzung dar. $\mathrm{KI}=$ Konfidenzintervall. $\mathrm{N}=1972$. 
Tabelle 5: Sensitivitätsanalyse für den Effekt auf den Erststimmenanteil

\begin{tabular}{|c|c|c|c|c|c|c|c|c|}
\hline & \multicolumn{4}{|c|}{ SPD } & \multicolumn{4}{|c|}{$\mathrm{CDU}$} \\
\hline & (1) & (2) & (3) & (4) & (1) & (2) & (3) & (4) \\
\hline Amtsinhaber & $\begin{array}{c}1,600 \\
(0,775)\end{array}$ & $\begin{array}{c}1,822 \\
(0,722)\end{array}$ & $\begin{array}{l}1,401 \\
(0,674)\end{array}$ & $\begin{array}{c}1,365 \\
(0,662)\end{array}$ & $\begin{array}{c}1,373 \\
(0,714)\end{array}$ & $\begin{array}{c}1,203 \\
(0,669)\end{array}$ & $\begin{array}{c}1,147 \\
(0,703)\end{array}$ & $\begin{array}{c}0,939 \\
(0,314)\end{array}$ \\
\hline Erststimmenanteil (t-1) & & $\begin{array}{c}0,532 \\
(0,031)\end{array}$ & $\begin{array}{c}5,461 \\
(1,005)\end{array}$ & $\begin{array}{c}4,924 \\
(1,003)\end{array}$ & & $\begin{array}{c}0,863 \\
(0,045)\end{array}$ & $\begin{array}{c}0,506 \\
(0,737)\end{array}$ & $\begin{array}{c}0,475 \\
(0,637)\end{array}$ \\
\hline 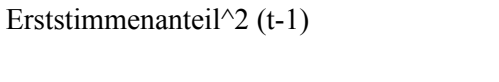 & & & $\begin{array}{c}0,007 \\
(0,004)\end{array}$ & $\begin{array}{c}0,004 \\
(0,004)\end{array}$ & & & $\begin{array}{c}0,017 \\
(0,011)\end{array}$ & $\begin{array}{c}0,032 \\
(0,006)\end{array}$ \\
\hline Zweitstimmenanteil (t-1) & & & $\begin{array}{l}-5,061 \\
(1,032)\end{array}$ & $\begin{array}{l}-4,924 \\
(1,026)\end{array}$ & & & $\begin{array}{c}0,567 \\
(0,717)\end{array}$ & $\begin{array}{c}0,056 \\
(0,656)\end{array}$ \\
\hline Zweitstimmenanteil^2 (t-1) & & & $\begin{array}{c}0,001 \\
(0,004)\end{array}$ & $\begin{array}{c}0,003 \\
(0,004)\end{array}$ & & & $\begin{array}{c}0,020 \\
(0,01)\end{array}$ & $\begin{array}{c}0,039 \\
(0,006)\end{array}$ \\
\hline Wahlbeteiligung (t-1) & & & $\begin{array}{l}1,364 \\
(0,476)\end{array}$ & $\begin{array}{c}1,550 \\
(0,464)\end{array}$ & & & $\begin{array}{l}-0,898 \\
(0,552)\end{array}$ & $\begin{array}{l}-0,928 \\
(0,311)\end{array}$ \\
\hline Wahlbeteiligung ${ }^{\wedge}(\mathrm{t}-1)$ & & & $\begin{array}{l}-0,007 \\
(0,003)\end{array}$ & $\begin{array}{l}-0,009 \\
(0,003)\end{array}$ & & & $\begin{array}{c}0,009 \\
(0,003)\end{array}$ & $\begin{array}{c}0,007 \\
(0,002)\end{array}$ \\
\hline Erststimmenanteil $\times$ Wahlbeteiligung & & & $\begin{array}{l}-0,077 \\
(0,012)\end{array}$ & $\begin{array}{l}-0,070 \\
(0,012)\end{array}$ & & & $\begin{array}{l}-0,006 \\
(0,008)\end{array}$ & $\begin{array}{l}-0,001 \\
(0,007)\end{array}$ \\
\hline Zweitstimmenanteil $\times$ Wahlbeteiligung & & & $\begin{array}{c}0,068 \\
(0,013)\end{array}$ & $\begin{array}{c}0,064 \\
(0,013)\end{array}$ & & & $\begin{array}{l}-0,006 \\
(0,008)\end{array}$ & $\begin{array}{c}0,000 \\
(0,008)\end{array}$ \\
\hline Erststimmenanteil $\times$ Zweitstimmenanteil & & & $\begin{array}{c}0,007 \\
(0,001) \\
\end{array}$ & $\begin{array}{c}0,009 \\
(0,001) \\
\end{array}$ & & & $\begin{array}{l}-0,028 \\
(0,021) \\
\end{array}$ & $\begin{array}{l}-0,070 \\
(0,011) \\
\end{array}$ \\
\hline
\end{tabular}

MM-Schätzungen mit robusten Standardfehlern in Klammern. Alle Schätzungen basieren auf einem multiplikativen Polynom vierten Grades gebildet aus der Zuweisungsvariable und dem Treatment-Indikator links u. rechts des Schwellenwertes. N = 197. 
Tabelle 6: Sensitivitätsanalyse für den Effekt auf den Zweitstimmenanteil

\begin{tabular}{|c|c|c|c|c|c|c|c|c|}
\hline & \multicolumn{4}{|c|}{ SPD } & \multicolumn{4}{|c|}{ CDU } \\
\hline & (1) & (2) & (3) & (4) & (1) & (2) & (3) & (4) \\
\hline \multirow[t]{2}{*}{ Amtsinhaber } & 1,491 & 1,755 & 1,043 & 0,922 & 1,057 & 0,946 & 0,898 & 0,551 \\
\hline & $(0,809)$ & $(0,767)$ & $(0,633)$ & $(0,626)$ & $(0,779)$ & $(0,680)$ & $(0,641)$ & $(0,287)$ \\
\hline \multirow{2}{*}{ Erststimmenanteil (t-1) } & & 0,482 & 5,019 & 4,105 & & 0,976 & 0,926 & 0,006 \\
\hline & & $(0,034)$ & $(1,141)$ & $(1,104)$ & & $(0,045)$ & $(0,719)$ & $(0,600)$ \\
\hline \multirow[t]{2}{*}{ Erststimmenanteil^2 (t-1) } & & & 0,004 & 0,000 & & & $-0,02$ & 0,001 \\
\hline & & & $(0,004)$ & $(0,004)$ & & & $(0,012)$ & $(0,010)$ \\
\hline \multirow[t]{2}{*}{ Zweitstimmenanteil (t-1) } & & & $-4,781$ & $-4,317$ & & & 0,361 & 0,642 \\
\hline & & & $(1,163)$ & $(1,122)$ & & & $(0,711)$ & $(0,626)$ \\
\hline \multirow[t]{2}{*}{ Zweitstimmenanteil ${ }^{\wedge} 2(\mathrm{t}-1)$} & & & 0,006 & 0,008 & & & $-0,012$ & 0,012 \\
\hline & & & $(0,004)$ & $(0,004)$ & & & $(0,011)$ & $(0,010)$ \\
\hline \multirow[t]{2}{*}{ Wahlbeteiligung (t-1) } & & & 0,946 & 1,240 & & & 0,251 & $-1,255$ \\
\hline & & & $(0,508)$ & $(0,482)$ & & & $(0,496)$ & $(0,273)$ \\
\hline \multirow[t]{2}{*}{ Wahlbeteiligung^2 (t-1) } & & & $-0,004$ & $-0,007$ & & & 0,003 & 0,009 \\
\hline & & & $(0,003)$ & $(0,003)$ & & & $(0,003)$ & $(0,002)$ \\
\hline \multirow[t]{2}{*}{ Erststimmenanteil $\times$ Wahlbeteiligung } & & & $-0,077$ & $-0,064$ & & & $-0,013$ & 0,004 \\
\hline & & & $(0,015)$ & $(0,015)$ & & & $(0,008)$ & $(0,007)$ \\
\hline \multirow[t]{2}{*}{ Zweitstimmenanteil $\times$ Wahlbeteiligung } & & & 0,068 & 0,059 & & & $-0,001$ & $-0,005$ \\
\hline & & & $(0,015)$ & $(0,015)$ & & & $(0,008)$ & $(0,007)$ \\
\hline \multirow[t]{2}{*}{ Erststimmenanteil $\times$ Zweitstimmenanteil } & & & 0,007 & 0,009 & & & 0,041 & $-0,010$ \\
\hline & & & $(0,001)$ & $(0,001)$ & & & $(0,023)$ & $(0,020)$ \\
\hline
\end{tabular}

MM-Schätzungen mit robusten Standardfehlern in Klammern. Alle Schätzungen basieren auf einem multiplikativen Polynom vierten Grades gebildet aus der Zuweisungsvariable und dem Treatment-Indikator links u. rechts des Schwellenwertes. N = 1972. 
Über verschiedene Spezifikationen hinweg sind unsere Schätzungen robust. Da sich die Befunde in beiden Tabellen gleichen, können sie gemeinsam diskutiert werden. Im Ausgangsmodell (1) wird der Treatment-Effekt (wie in Tabelle 1) ohne Berücksichtigung weiterer Variablen geschätzt. Modell 2 kontrolliert für den Erststimmenanteil bei der vorhergehenden Wahl. Die Größe des Treatment-Effekts ändert sich nicht merklich; die Präzision unserer Schätzung ist etwas höher. Der Zweitstimmenanteil, die Wahlbeteiligung der vorhergehenden Wahl und die quadrierten Werte und Interaktionsterme dieser Variablen werden in Modell 3 aufgenommen. Der Treatment-Effekt bleibt nahezu unverändert. Abschließend kontrollieren wir für Bundesland- und Jahreseffekte in Modell 4. Der Koeffizient für den Amtsinhaberstatus nimmt leicht ab, wird aber umso präziser geschätzt. Auch für die CDU/CSU wird damit ein konventionelles Signifikanzniveau erreicht.

\section{Fazit und Ausblick}

Die Amtsinhaberschaft einer Partei wird in dieser Arbeit als neue Quelle von Kontaminationseffekten in kombinierten Wahlsystemen identifiziert. Unser Argument stützt sich auf die Verbindung der klassischen Literatur zur Wirkung des Amtsinhaberstatus mit den neueren Arbeiten zu Kontaminationseffekten. Wenn sich die bloße Beteiligung im majoritären Wahlsystemelement in Form der Aufstellung eines Wahlkreisbewerbers positiv auf den Zweitstimmenanteil einer Partei auswirkt, dann sollte der Amtsinhaberstatus einer Partei einen noch stärkeren Einfluss besitzen. Diese Hypothese wird durch die Daten nicht falsifiziert. Die Amtsinhaberschaft erhöht den Zweitstimmenanteil einer Partei um 1 bis 1,5 Prozentpunkte. Die Simulationen zeigen, dass dies ausreichend sein kann, um die Mehrheitsverhältnisse im Bundestag entscheidend zu ändern. Ebenso bestätigen unsere Ergebnisse die Vermutung vorheriger Arbeiten, dass sich die Amtsinhaberschaft auch positiv auf den Erststimmenanteil einer Partei auswirkt.

Unsere Befunde haben sowohl für die Kontaminationsforschung als auch für die dem strategischen Verhalten deutscher Parteien gewidmete Literatur erhebliche Bedeutung. Mit Blick auf die Forschung zu Kontaminationseffekten ist erstens festzuhalten, dass unsere Ergebnisse die Behauptung von Ferrara et al. (2005) bestätigen, kombinierte Wahlsysteme ließen keinen kontrollierten Vergleich von Wählerverhalten unter den Bedingungen unterschiedlicher Wahlsysteme zu (Moser und Scheiner 2005: 260). Zwischen Erst- und Zweitstimmen 
besteht in der Tat eine erhebliche Wechselwirkung, so dass diese beiden Elemente in kombinierten Wahlsystemen nicht als unabhängige Beobachtungen betrachtet und analysiert werden können.

Zweitens sind in Zukunft jene kausalen Mechanismen zu untersuchen, die den hier ausgemachten Kontaminationseffekten der Amtsinhaberschaft zugrunde liegen. Ist die erhöhte Medienpräsenz und Bekanntheit des Wahlkreisabgeordneten oder vor allem der von ihm geleistete Wähler- und Wahlkreisservice für den höheren Zweitstimmenanteil verantwortlich? Mittels der in dieser Arbeit verwendeten Aggregatdaten vermögen wir nicht zwischen diesen möglichen Erklärungen zu diskriminieren. Hierfür bedürfte es detaillierter Paneldaten auf Individualebene, die nach unserem Wissen noch nicht existieren.

Eine interessante Implikation unserer Arbeit für die dem strategischen Verhalten deutscher Parteien gewidmete Literatur ist, dass Parteigremien die Qualität der Wahlkreisarbeit bzw. die Medienpräsenz als wichtiges Kriterium bei der Kandidatenaufstellung berücksichtigen sollten. Obwohl eine quantitative Untersuchung dieser Frage nach wie vor aussteht, deutet einiges darauf hin, dass sowohl SPD als auch CDU/CSU die erwartete Qualität des Wählerservice in ihre Entscheidungen über die Aufstellung von Wahlkreisbewerbern einfließen lassen (Zeuner 1970: 144-6; Patzelt 1993: 366-82). Die bisherige Literatur hat dieser Beobachtung jedoch keine besondere Aufmerksamkeit geschenkt. Unsere Ergebnisse legen nahe, dass sich die Berücksichtigung der erwarteten Qualität der Wahlkreisarbeit angesichts von Kontaminationseffekten als ein rationaler Akt der Zweitstimmenmaximierung auffassen lässt.

Eine weitere Implikation unserer Arbeit ergibt sich für kleinere Parteien. Bislang wurde in der Literatur argumentiert, dass sich gerade diese durch das Aufstellen eines eigenen (wenn auch oft hoffungslosen) Wahlkreisbewerbers auf Kosten der großen Parteien profilieren können. Unsere Befunde legen dahingegen nahe, dass Kontaminationseffekte auch in die entgegengesetzte Richtung wirken können. Da der Amtsinhaberstatus einer Partei positiv auf ihren Zweitstimmenanteil wirkt und üblicherweise nur Kandidaten von SPD oder CDU/CSU Direktmandate erringen, könnten die hier identifizierten Effekte diesen Vorteil kleinerer Parteien wieder zunichte machen. Leider limitiert das Forschungsdesign unsere Untersuchung insofern, als Veränderungen über Zeit nicht analysiert werden können. Die nachlassende Parteibindung der Wähler (Dalton und Wattenberg 2000) gepaart mit der zunehmenden Personalisierung der Politik (Pappi 1999; von Alemann 2003: 191-203) lässt jedoch vermuten, dass Amtsinhabereffekte an Stärke zunehmen werden. Es erscheint plausibel, dass dies auch den Anreiz weiter verstärken wird, Wahlkreisbewerber mit aller 
Sorgfalt insbesondere im Hinblick auf den von ihnen zu erwartenden Wahlkreisund Wählerservice auszuwählen.

\section{Literatur}

Angrist, Joshua und Victor Lavy, 1999: Using Maimonides' Rule to Estimate the Effect of Class Size on Scholastic Achievement. Quarterly Journal of Economics 114, 533-567.

Ansolabehere, Stephen und James M. Snyder, 2004: Using Term Limits to Estimate Incumbency Advantages When Officeholders Retire Strategically. Legislative Studies Quarterly XXIX, 487-515.

Ansolabehere, Stephen, James M. Snyder und Charles Stewart, 2000. Old Voters, New Voters, and the Personal Vote: Using Redistricting to Measure the Incumbency Advantage. American Journal of Political Science 44, 17-34.

Battistin, Erich und Enrico Rettore, 2002: Testing for Program Effects in a Regression Discontinuity Design with Imperfect Compliance. Journal of the Royal Statistical Society, Series A 165, 39-57.

Battistin, Erich und Enrico Rettore, 2003: Another Look at the Regression Discontinuity Design. Working Paper, Institute for Fiscal Studies, London.

Bawn, Kathleen, 1993: The Logic of Institutional Preferences: German Electoral Law as a Social Choice Outcome. American Journal of Political Science 37, 965-989.

Bawn, Kathleen, 1999: Voter Responses to Electoral Complexity: Ticket Splitting, Rational Voters and Representation in the Federal Republic of Germany. British Journal of Political Science 28, 487-505.

Bawn, Kathleen und Michael F. Thies, 2003: A Comparative Theory of Electoral Incentives: Representing the Unorganized Under PR, Plurality, and Mixed-Member Electoral Systems. Journal of Theoretical Politics 15, 5-32.

Berk, Richard A. und Jan de Leeuw, 1999: An Evaluation of Californias Inmate Classification System Using a Generalized Regression Discontinuity Design. Journal of the American Statistical Association 94, 1045-1052.

Black, Sandra E., 1999: Do Better Schools Matter? Parental Valuation of Elementary Education. Quarterly Journal of Economics 144, 577-599.

Brettschneider, Frank, 2002: Spitzenkandidaten und Wahlerfolg. Personalisierung Kompetenz - Parteien. Wiesbaden: VS Verlag.

Butler, Daniel. M. und Matthew J. Butler, 2005: Splitting the Difference: What Explains Split-party Delegations in the US Senate? Working paper, Stanford University.

Caramani, Daniele, 2000: Elections in Western Europe since 1815: Electoral Results by Constituencies. London: Palgrave Macmillan.

Cox, Gary W., 1997: Making Votes Count: Strategic Coordination in the Worlds Electoral Systems. Cambridge: Cambridge University Press.

Cox, Gary W. und Jonathan N. Katz, 2002: Elbridge Gerry's Salamander. Cambridge, New York: Cambridge University Press. 
Cox, Karen E. und Leonard J. Schoppa, 2002: Interaction Effects in Mixed-Member Electoral Systems. Comparative Political Studies 35, 1027-1053.

Croux, Christophe, Geert Dhaene und Dirk Hoorelbeke, 2003: Robust Standard Errors for Robust Estimators. Research Report, Dept. of Applied Economics, K.U. Leuven.

Dalton, Russel J. und Martin P. Wattenberg, 2000: Parties without Partisans: Political Change in Advanced Industrial Democracies. Oxford: Oxford University Press.

DiNardo, John und David S. Lee, 2004: Economic Impacts of New Unionization on Private Sector Employers: 1984-2001. Quarterly Journal of Economics 119, 13831442.

Duverger, Maurice, 1986: Duverger's Law: Forty Years Later. In: Bernard Grofman und Arend Lijphart (Hrsg.), Electoral laws and their political consequences, 80-84, New York: Agathon.

Duverger, Maurice, 1954: Political Parties, their Organization and Activity in the Modern State. New York: Wiley.

Falter, Jürgen, Oscar W. Gabriel und Hans Rattinger, 2000: Wirklich ein Volk?: Die Politischen Orientierungen von Ost- und Westdeutschen im Vergleich. Opladen: Leske+Budrich.

Ferrara, Federico, Erik S. Herron und Misa Nishikawa, 2005: Mixed Electoral Systems: Contamination and its Consequences. New York: Palgrave Macmillan.

Gelman, Andrew und Gary King, 1990: Estimating Incumbency Advantage without Bias. American Journal of Political Science 34, 1142-64.

Grofman, Bernard und Arendt Lijphart. 1986: Electoral Laws and their Political Consequences. New York: Agathon Press.

Gschwend, Thomas, Ron Johnston und Charles Pattie, 2003: Split-ticket Patterns in Mixed-Member Proportional Election Systems: Estimates and Analyses of their Spatial Variation at the German Federal Election, 1998. British Journal of Political Science 33, 109-127.

Hahn, Jinyong, Petra Todd und Wilbert van der Klaauw, 2001: Identification and Estimation of Treatment Effects with a Regression-Discontinuity Design. Econometrica 69, 201-209.

Herron, Erik S. und Misa Nishikawa, 2001: Contamination Effects and the Number of Parties in Mixed-Superposition Electoral Systems. Electoral Studies 20, 63-86.

Herzog, Dietrich, Hilke Rebensdorf, Camilla Werner, und Bernhard Wesseles, 1990: Abgeordnete und Buerger. Opladen: Westdeutscher Verlag.

Holland, Paul W., 1986: Statistics and Causal Inference. Journal of the American Statistical Association 81, 945-960.

Hough, Daniel und Charlie Jeffrey, 2003: Landtagswahlen: Bundestestwahlen oder Regionalwahlen? Zeitschrift für Parlamentsfragen 34, 79-94.

Jeffery, Charlie und Daniel Hough, 2001: The Electoral Cycle and Multi-level Voting in Germany. German Politics 10, 73-98.

Kern, Holger Lutz und Jens Hainmueller, im Erscheinen. Electoral Balancing, Divided Government, and 'Midterm' Loss in German Elections. Journal of Legislative Studies. 
King, Gary und Andrew Gelman, 1991: Systemic Consequences of Incumbency Advantage in U.S. House Elections. American Journal of Political Science 35, 110-138.

Klingemann, Hans-Dieter und Bernhard Wessels, 2001. The Political Consequences of Germany's Mixed-Member System: Personalization at the Grass Roots? In: Matthew S. Shugart und Martin P. Wattenberg (Hrsg.), Mixed-Member Electoral Systems: The Best of Both Worlds?, 279-297, Oxford: Oxford University Press.

Knack, Steve, 1994: Does Rain Help the Republicans? Theory and Evidence on Turnout and the Vote. Public Choice 79, 187-209.

Lancaster, Thomas und W. David Patterson, 1990: Comparative Pork Barrel Politics: Perceptions from the West German Bundestag. Comparative Political Studies 22, 458-477.

Lee, David S., im Erscheinen: Randomized Experiments from Non-random Selection in U.S. House Elections. Journal of Econometrics.

Lee, David S. und David Card, 2004: Regression Discontinuity Inference with Specification Error. Working paper, UC Berkeley.

Lee, David S., Enrico Moretti und Matthew J. Butler, 2004: Do Voters Affect or Elect Policies? Evidence from the U.S. House. Quarterly Journal of Economics 119, 807859.

Levitt, Steven D. und Catherine D. Wolfram, 1997: Decomposing the Sources of Incumbency Advantage in the U.S. House. Legislative Studies Quarterly XXII, 45-60.

Martorell, Francisco, 2004: Do Graduation Exams Matter? A Regression-Discontinuity Analysis of the Impact of Failing the Exit Exam on High School and Post-High School Outcomes. Working paper, UC Berkeley.

Massicotte, Louis und Andre Blais, 1999: Mixed Electoral Systems: A Conceptual and Empirical Survey. Electoral Studies 18, 244-366.

Matsudaira, Jordan D., 2004: Sinking or Swimming? Evaluating the Impact of English Immersion vs. Bilingual Education on Student Achievement. Working paper, University of Michigan.

Matsusaka, John G. und Filip Palda, 1999: Voter Turnout: How Much Can We Explain? Public Choice 98, 431-446.

Moser, Robert G., 1999: Electoral Systems and the Number of Parties in Post-Communist States. World Politics 51, 359-384.

Moser, Robert G. und Ethan Scheiner, 2005: Strategic Ticket Splitting and the Personal Vote in Mixed-Member Electoral Systems. Journal of Legislative Studies 30, 259276.

Nohlen, Dieter, 2004: Wahlrecht und Parteiensystem. Opladen: Leske + Budrich.

Pappi, Franz Urban und Susumu Shikano (2001): Personalisierung der Politik in Mehrparteiensystemen am Beispiel deutscher Bundestagswahlen seit 1980. Politische Vierteljahresschrift 42, 355-387.

Patzelt, Werner J., 1993: Abgeordnete und Repräsentation: Amtsverständnis und Wahlkreisarbeit. Passau: Wissenschaftsverlag Rothe.

Porter, Jack, 2002: Asymptotic Bias and Optimal Convergence Rates for Semi-parametric Kernel Estimators in the Regression Discontinuity Model. Working Paper, Harvard Institute of Economic Research Discussion paper no. 1989.

Rosenbaum, Paul R., 2002: Observational Studies, 2nd edn, New York: Springer-Verlag. 
Rousseeuw, Peter und Victor J. Yohai, 1984: Robust Regression by means of Sestimators. S. 256-272 in: Jürgen Franke, Wolfgang Härdle, und D. Martin (editors): Robust and Nonlinear Time Series Analysis. Lecture Notes in Statistics No. 26. Berlin: Springer-Verlag.

Rubin, Donald B., 1974: Estimating Causal Effects of Treatments in Randomized and Nonrandomized Studies. Journal of Educational Psychology 66, 688-701.

Rubin, Donald B., 1977. Assignment to Treatment Group on the Basis of a Covariate. Journal of Educational Statistics 2, 1-26.

Rubin, Donald B., 1978: Bayesian Inference for Causal Effects: The Role of Randomization. Annals of Statistics, 6, 34-58.

Rubin, Donald B., 1990: Comment: Neyman (1923) and Causal Inference in Experiments and Observational Studies. Statistical Science 5, 472-480.

Shadish, William R., Thomas D. Cook und Donald T. Campbell, 2002: Experimental and Quasi-Experimental Designs for Generalized Causal Inference. Boston: HoughtonMifflin.

Shugart, Matthew S., 2001: Electoral Efficiency and the Move to Mixed-Member Systems. Electoral Studies 20, 173-193.

Shugart, Matthew S. und Martin P. Wattenberg, 2001: Mixed-Member Electoral Systems: The Best of Both Worlds? Oxford: Oxford University Press.

Sobel, Michael E., 2003: What do Randomized Studies of Housing Mobility Reveal? Causal Inference in the Face of Interference. Working paper, Columbia University.

Stratmann, Thomas und Martin Baur, 2002: Plurality Rule, Proportional Representation, and the German Bundestag: How Incentives to Pork-Barrel Differ Across Electoral Systems. American Journal of Political Science 46, 506-514.

Thistlethwaite, Donald und Donald Campbell, 1960: Regression-Discontinuity Analysis: An Alternative to the Ex Post Facto Experiment. Journal of Educational Psychology $51,309-17$.

Trochim, William, 1984: Research Design for Program Evaluation: The Regression Discontinuity Approach. Beverly Hills: Sage Publications.

von Alemann, Ulrich, 2003: Das Parteiensystem der Bundesrepublik Deutschland. Opladen: Leske \& Budrich.

Woyke, Wichard, 1994: Stichwort: Wahlen, 8th edn. Opladen: Leske + Budrich.

Yohai, Victor J., 1987: High breakdown point and high efficiency robust estimates for regression. Annals of Statistics 15, 642-656.

Yohai, Victor J., Werner Stahel und Ruben H. Zamar, 1991: A procedure for robust estimation and inference in linear regression. S. 35-49 in: Werner Stahel, und Sanford Weisberg (Hrsg.): Directions in Robust Statistics and Diagnostics, Part II. New York: Springer-Verlag.

Zeuner, Bodo, 1970: Kandidatenaufstellung zur Bundestagswahl 1965. Den Haag: Martinus Nijhoff. 